\title{
Optimal full-waveform inversion strategy for marine data in azimuthally rotated elastic orthorhombic media
}

\author{
Ju-Won $\mathrm{Oh}^{1}$ and Tariq Alkhalifah ${ }^{2}$
}

\begin{abstract}
The orthorhombic (ORT) anisotropic description of earth layers can allow the capture of much of the earth's anisotropic complexity. The inversion for high-resolution azimuthal variation of anisotropy is important for reservoir characterization, among other applications. A high-resolution description of the azimuth of fractures can help us to predict flow preferences. To verify the feasibility of multiparameter full-waveform inversion (FWI) for marine data assuming azimuthally rotated elastic ORT media, we have analyzed the radiation patterns and gradient directions of ORT parameters to the reflection data. First, we express the gradient direction of the ORT parameters considering the azimuthal rotation of the symmetric planes. Then, to support our observations in the gradient direction, the radiation patterns of the partial derivative wavefields from each parameter
\end{abstract}

perturbation are also derived under the rotated elastic ORT assumption. To find an optimal parameterization, we compare three different parameterizations: monoclinic, velocity-based, and hierarchical parameterizations. Then, we suggest an optimal multistage update strategy by analyzing the behavior of the rotation angle as a FWI target. To analyze the trade-off among parameters in different parameterizations, we calculate the gradient direction from a hockey-puck model, in which each parameter is perturbed at the different location on a horizontal layer. The trade-off analysis supports that the hierarchical parameterization provides us with more opportunities to build up subsurface models with less trade-off between parameters and less influence of the azimuthal rotation of ORT anisotropy. The feasibility of the proposed FWI strategy is examined using synthetic marine streamer data from a simple 3D reservoir model with a fractured layer.

\section{INTRODUCTION}

Full-waveform inversion (FWI) is a seismic interpretation technique that estimates subsurface physical properties from the seismic data through mathematical optimization procedures without drilling the earth (Vireiux and Operto, 2009). The physical properties can be wave velocities, anisotropic parameters, viscosity, and so on depending on the FWI purpose. Considering such opportunities, the application of multiparameter FWI in exploration seismology has gained momentum over the past decade.

In multiparameter FWI, one important issue is finding an optimal parameterization to represent the model (Operto et al., 2013). Many geophysicists, using various physical assumptions, have carried out parameterization analysis over the years. The multiparameter FWI analysis has been presented for the isotropic, variable density, acoustic case (Operto et al., 2013; Bai and Yingst, 2014; Ma et al., 2016; Oh and Alkhalifah, 2018), and elastic earth (Tarantola, 1986; Brossier et al., 2009; Köhn et al., 2012, 2015; Prieux et al., 2013; Raknes and Weibull, 2016; Oh et al., 2018). Considering that anisotropy is prevalent inside the earth, multiparameter FWI for anisotropic media has gotten plenty of attention as an ingredient to help convergence of FWI. The vertical transverse isotropic (VTI) (Tsvankin, 2012) model is the simplest anisotropic model, which includes the intrinsic anisotropy within sedimentary layers and the apparent anisotropy caused by thin horizontal layering (Backus, 1962). The optimal parameterization for acoustic VTI media (Gholami et al., 2013; Alkhalifah and Plessix, 2014; He and Plessix, 2016; Silva et al., 2016) and elastic VTI media (Oh et al., 2015;

Manuscript received by the Editor 20 November 2017; revised manuscript received 21 January 2018; published ahead of production 09 March 2018; published online 8 June 2018.

${ }^{1}$ Formerly King Abdullah University of Science and Technology, Physical Science and Engineering Division, 4700, Thuwal 23955-6900, Kingdom of Saudi Arabia; presently Chonbuk National University, Department of Resources and Energy Engineering, 567, Baekje-daero, Deokjin-gu, Jeonju-si, Jeollabuk-do 54896, Republic of Korea. E-mail: juwon24@jbnu.ac.kr.

${ }^{2}$ King Abdullah University of Science and Technology, Physical Science and Engineering Division, 4700, Thuwal 23955-6900, Kingdom of Saudi Arabia. E-mail: tariq.alkhalifah@ kaust.edu.sa.

(C) 2018 Society of Exploration Geophysicists. All rights reserved. 
Kamath and Tsvankin, 2016) has been studied. Another simple anisotropic model is the horizontal transverse isotropic (HTI) model, which includes vertically aligned fracture networks (Tsvankin, 2012). Pan et al. (2016) examine the Gauss-Newton approach to invert for HTI parameters.

To take the VTI and HTI models into account, we describe the earth using an orthorhombic (ORT) anisotropic model (Tsvankin, 1997; Stovas, 2015). Although the study on ORT FWI has been rarely conducted due to the high computational cost of such an endeavor, recent developments in supercomputing allow us to work on FWI for this complex anisotropic model (Albertin et al., 2016; Masmoudi and Alkhalifah, 2016; Oh and Alkhalifah, 2016a, 2016b; Wang and Tsvankin, 2016; Xie et al., 2017).

However, in such an ORT model, we have often ignored the role that the symmetry azimuth direction plays in such an inversion. The symmetry azimuth holds crucial information on tectonic (stress) behavior of the region and more importantly fracture direction information in a potential reservoir. Conventional approaches to estimate fracture direction (Bakulin et al., 2000) rely on amplitude versus offset and azimuth inversion (Downton and Roure, 2010; Yin et al., 2013). Such approaches have a 1D preference and fail in more complex media, where lateral inhomogeneity is prevalent. With FWI, we can handle more complex media, and thus, we have an opportunity to invert for ORT azimuth in such a medium. However, most inversion approaches rely on a monoclinic (Rusmanugroho et al., 2017) or a triclinic model (Köhn et al., 2015). Because monoclinic media have more independent parameters to be inverted compared with rotated anisotropic media, a rotated ORT model is more practical if the rotation angle information is available.

In this study, we examine the possibility of inverting for the rotation angle of the symmetric axis (i.e., fracture direction) through the FWI procedure. To do that, we first formulate the azimuthally rotated elastic ORT FWI. Then, to find an optimal strategy to invert for the rotation angle, we find the most optimal parameterization and an optimal update strategy by comparing three different parameterizations: monoclinic, velocity-based, and hierarchical parameterizations through their radiation-pattern and gradient analyses. Based on the observations in the radiation-pattern analysis, we suggest a multistage FWI strategy using the hierarchical parameterization. To support our observations, the trade-off analysis for velocity-based and hierarchical parameterizations is conducted assuming a simple hockey-puck model. Finally, the proposed multistage strategy is examined by the marine streamer data from a synthetic 3D model with a fractured reservoir.

\section{INVERSE THEORY}

\section{Inverse problem in rotated elastic orthorhombic media}

The goal of FWI is finding the global minimum solution to minimize the data misfits between observed data $d$ and modeled data $u$. The $l_{2}$-norm, the most commonly used objective function, can be expressed as follows (Tarantola, 1986):

$$
E(\mathbf{m})=\sum_{s} \sum_{r}[u(s, r, \mathbf{m})-d(s, r)]^{2},
$$

where $s$ and $r$ indicate the source and receiver indices, respectively. Depending on the physical model assumed in the simulation (ignoring viscosity), the model parameter vector $\mathbf{m}$ could include from 1 (monoparameter acoustic media) to 22 parameters (triclinic elastic media) including the density. As we consider a more complex physical model, we will need more parameters to describe the physical phenomena.

The elastic wave equation for a given seismic source $f_{i}$ is expressed here in terms of particle displacements $u_{i}$, stress $\sigma_{i j}$, and strain $\varepsilon_{k l}$ as follows (Tsvankin, 2001):

$$
\rho \frac{\partial^{2} u_{i}}{\partial^{2} t}=\partial_{j} \sigma_{i j}+f_{i}
$$

and

$$
\sigma_{i j}=\tilde{C}_{i j k l} \varepsilon_{k l}
$$

where the parameter $\rho$ is the density. In this study, the density is ignored and is not updated during FWI (Guitton and Alkhalifah, 2016). The term $\tilde{C}_{i j k l}$ denotes the elastic constants. For the azimuthally rotated elastic ORT media, the stiffness matrix contains 13 $\tilde{C}_{I J}$ (in Voigt notation) nonzero parameters. In contrast to monoclinic media represented by 13 independent parameters, the rotated ORT media is represented by 10 independent parameters, including nine ORT parameters and the rotation angle $\varphi$ of symmetry axis. This conversion can be expressed using the Bond transformation around the vertical $z$-axis in a counterclockwise direction (Ivanov and Stovas, 2016):

$$
\mathbf{C}_{I J}^{r \mathrm{ORT}}=\mathbf{D}_{z}(\phi) \mathbf{C}_{I J}^{r \mathrm{ORT}} \mathbf{D}_{z}^{T}(\phi),
$$

where

$$
\begin{aligned}
\mathbf{C}^{r \mathrm{ORT}} & =\left(\begin{array}{cccccc}
\tilde{C}_{11} & \tilde{C}_{12} & \tilde{C}_{13} & 0 & 0 & \tilde{C}_{16} \\
\tilde{C}_{12} & \tilde{C}_{22} & \tilde{C}_{23} & 0 & 0 & \tilde{C}_{26} \\
\tilde{C}_{13} & \tilde{C}_{23} & \tilde{C}_{33} & 0 & 0 & \tilde{C}_{36} \\
0 & 0 & 0 & \tilde{C}_{44} & \tilde{C}_{45} & 0 \\
0 & 0 & 0 & \tilde{C}_{45} & \tilde{C}_{55} & 0 \\
\tilde{C}_{16} & \tilde{C}_{26} & \tilde{C}_{36} & 0 & 0 & \tilde{C}_{66}
\end{array}\right), \\
\mathbf{C}^{\mathrm{ORT}} & =\left(\begin{array}{cccccc}
C_{11} & C_{12} & C_{13} & 0 & 0 & 0 \\
C_{12} & C_{22} & C_{23} & 0 & 0 & 0 \\
C_{13} & C_{23} & C_{33} & 0 & 0 & 0 \\
0 & 0 & 0 & C_{44} & 0 & 0 \\
0 & 0 & 0 & 0 & C_{55} & 0 \\
0 & 0 & 0 & 0 & 0 & C_{66}
\end{array}\right),
\end{aligned}
$$

and

$$
\mathbf{D}_{z}(\phi)=\left(\begin{array}{cccccc}
\cos ^{2} \phi & \sin ^{2} \phi & 0 & 0 & 0 & -2 \sin \phi \cos \phi \\
\sin ^{2} \phi & \cos ^{2} \phi & 0 & 0 & 0 & 2 \sin \phi \cos \phi \\
0 & 0 & 1 & 0 & 0 & 0 \\
0 & 0 & 0 & \cos \phi & \sin \phi & 0 \\
0 & 0 & 0 & -\sin \phi & \cos \phi & 0 \\
\sin \phi \cos \phi & -\sin \phi \cos \phi & 0 & 0 & 0 & \cos ^{2} \phi-\sin ^{2} \phi
\end{array}\right) .
$$

As a result, $13 \tilde{C}_{I J}$ parameters in equation 5 are expressed as follows: 


$$
\left(\begin{array}{l}
\tilde{C}_{11} \\
\tilde{C}_{22} \\
\tilde{C}_{33} \\
\tilde{C}_{12} \\
\tilde{C}_{13} \\
\tilde{C}_{23} \\
\tilde{C}_{44} \\
\tilde{C}_{55} \\
\tilde{C}_{66} \\
\tilde{C}_{16} \\
\tilde{C}_{26} \\
\tilde{C}_{36} \\
\tilde{C}_{45}
\end{array}\right)=\left(\begin{array}{c}
C_{11} \cos ^{4} \phi+C_{22} \sin ^{4} \phi+2\left(C_{12}+2 C_{66}\right) \sin ^{2} \phi \cos ^{2} \phi \\
C_{11} \sin ^{4} \phi+C_{22} \cos ^{4} \phi+2\left(C_{12}+2 C_{66}\right) \sin ^{2} \phi \cos ^{2} \phi \\
C_{33} \\
C_{12}\left(\sin ^{4} \phi+\cos ^{4} \phi\right)+\left(C_{11}+C_{22}-4 C_{66}\right) \sin ^{2} \phi \cos ^{2} \phi \\
C_{13} \cos ^{2} \phi+C_{23} \sin ^{2} \phi \\
C_{13} \sin ^{2} \phi+C_{23} \cos ^{2} \phi \\
C_{44} \cos ^{2} \phi+C_{55} \sin ^{2} \phi \\
C_{44} \sin ^{2} \phi+C_{55} \cos ^{2} \phi \\
\left(C_{11}-C_{12}-2 C_{66}\right) \sin \phi \cos ^{3} \phi-\left(C_{22}-C_{12}-2 C_{66}\right) \cos \phi \sin ^{3} \phi \\
\left(C_{11}-C_{12}-2 C_{66}\right) \cos \phi \sin ^{3} \phi-\left(C_{22}-C_{12}-2 C_{66}\right) \sin \phi \cos ^{3} \phi \\
\left(C_{13}-C_{23}\right) \sin \phi \cos \phi \\
\left(C_{55}-C_{44}\right) \sin \phi \cos \phi
\end{array}\right) .
$$

\section{Gradient direction from monoclinic parameters}

The gradient direction for each parameter, required to minimize the objective function, can be obtained by taking the partial derivative of equation 1 with respect to that model parameter. In this procedure, taking the partial derivative of the elastic wave equations, we obtain the equations for the partial derivative wavefields (PDWs) of an arbitrary parameter perturbation $m$ at $n$th grid location as the same form as equation 2 :

$$
\rho \frac{\partial^{2} u_{\mathrm{PDW}, i}^{n}}{\partial^{2} t}=\partial_{j} \sigma_{\mathrm{PDW}, i j}^{n}+\partial_{j}\left(\frac{\partial \tilde{C}_{i j k l}}{\partial m_{n}} \varepsilon_{k l}\right),
$$

where

$$
u_{\mathrm{PDW}, i}^{n}=\frac{\partial u_{i}}{\partial m_{n}}
$$

and

$$
\sigma_{\mathrm{PDW}, i j}^{n}=\frac{\partial \sigma_{i j}}{\partial m_{n}}
$$

The last term in equation 9 represents the virtual source for each model parameter perturbation (Pratt et al., 1998). Based on the adjoint form, the gradient direction for each $\tilde{C}_{i j k l}$ parameter is expressed by (Kamath and Tsvankin, 2016)

$$
\nabla_{\tilde{C}_{i j k l}} E=\int_{0}^{T} \partial_{j} u_{i}^{s} \partial_{l} u_{k}^{r} d t,
$$

where $u^{s}$ and $u^{r}$ are the source and back-propagated residual wavefields (Pratt et al., 1998). The gradient direction for any parameter $m$ can be calculated using the chain rule as follows:

$$
\nabla_{m} E=\sum_{i j k l} \frac{\partial \tilde{C}_{i j k l}}{\partial m}\left(\nabla_{\tilde{C}_{i j k l}} E\right) .
$$

\section{Radiation-pattern analysis considering azimuthal rotation}

The radiation patterns of the PDW are instructive interpretation tools in multiparameter FWI, as they provide some insights on the trade-off between the parameters (Gholami et al., 2013; Alkhalifah and Plessix, 2014). Because the gradient direction of each parameter is determined by the zero-lag crosscorrelation between PDW and residual wavefields (Operto et al., 2013), the radiation pattern of the PDW for each parameter determines which part of data contribute in recovering that parameter. Based on the definition of angle in the radiation-pattern plot, the diffraction patterns (Tarantola, 1986; Pan et al., 2016; Rusmanugroho et al., 2017), the reflection patterns (Gholami et al., 2013; Alkhalifah and Plessix, 2014; Oh and Alkhalifah, 2016a), and the transmission patterns (Alkhalifah et al., 2016; He and Plessix, 2016; Kamath and Tsvankin, 2016) are used to find an optimal parameterization that has less trade-off among parameters. Because we focus on reflections from predominately horizontal and the diving waves from velocity variations along the vertical direction, we rely on the reflection (for a horizontal reflector) patterns in this paper.

The angular dependency of PDW from the incidence P-wave in an isotropic background medium can be approximated as follows (Aki and Richards, 1980; Oh and Alkhalifah, 2016a; Pan et al., 2016):

$$
\mathbf{u}_{\mathrm{PDW}(\mathrm{m})}^{\mathrm{P}-\mathrm{P}}\left(\theta_{i}, \varphi_{i}, \theta_{d}, \varphi_{d}\right)=\left[\mathbf{e}_{P_{d}}^{T} \mathbf{M}_{m}^{P_{i}} \mathbf{e}_{P_{d}}\right] \mathbf{e}_{P_{d}}=R_{m}^{\mathrm{P}-\mathrm{P}} \mathbf{e}_{P_{d}}
$$

and

$$
\mathbf{u}_{\mathrm{PDW}(\mathrm{m})}^{\mathrm{P}-\mathrm{SV}}\left(\theta_{i}, \varphi_{i}, \theta_{d}, \varphi_{d}\right)=\left[\mathbf{e}_{\mathrm{SV}_{d}}^{T} \mathbf{M}_{m}^{\mathrm{P}_{\mathrm{i}}} \mathbf{e}_{P_{d}}\right] \mathbf{e}_{\mathrm{P}_{\mathrm{d}}}=R_{m}^{\mathrm{P}-\mathrm{SV}} \mathbf{e}_{P_{d}},
$$

where

$$
\mathbf{e}_{P_{d}}^{T}=\left(\begin{array}{lll}
\sin \theta_{d} \cos \varphi_{d} & \sin \theta_{d} \sin \varphi_{d} & \cos \theta_{d}
\end{array}\right)
$$

and

$$
\mathbf{e}_{\mathrm{SV}_{d}}^{T}=\left(\begin{array}{lll}
\cos \theta_{d} \cos \varphi_{d} & \cos \theta_{d} \sin \varphi_{d} & -\sin \theta_{d}
\end{array}\right) .
$$

The four angles, $\theta_{i}, \theta_{d}, \varphi_{i}$, and $\varphi_{d}$, denote the incidence, diffraction, and azimuth angles of the incidence and diffraction planes, respectively. The incidence and diffraction angles are measured clockwise from the vertical axis, whereas the azimuth angles are defined by a counterclockwise angle from inline direction. Matrix $\mathbf{M}$ is the moment tensor form of the virtual source (Pratt et al., 1998) for each parameter listed in Table 1. The strains caused by the incidence P-wave can be approximated by (Pan et al., 2016)

$$
\left(\begin{array}{c}
\varepsilon_{x} \\
P_{i} \\
\varepsilon_{y} \\
P_{i} \\
\varepsilon_{z} \\
\varepsilon_{y z} \\
P_{i} \\
\varepsilon_{x z} \\
\varepsilon_{x y}
\end{array}\right)=\left(\begin{array}{c}
\sin ^{2} \theta_{i} \cos ^{2} \varphi_{i} \\
\sin ^{2} \theta_{i} \sin ^{2} \varphi_{i} \\
\cos ^{2} \theta_{i} \\
\sin \theta_{i} \cos \theta_{i} \sin \varphi_{i} \\
\sin \theta_{i} \cos \theta_{i} \cos \varphi_{i} \\
\sin \theta_{i} \sin \varphi_{i} \cos \varphi_{i}
\end{array}\right) .
$$

The terms $R_{m}^{\mathrm{P}-\mathrm{P}}$ and $R_{m}^{\mathrm{P}-\mathrm{SV}}$ indicate the P-P and P-SV diffraction patterns caused by a perturbation of the model parameter $m$. If we consider all 3C components or pressure data, the polarization vector $\mathbf{e}_{P_{d}}$ in equations 14 and 15 can be ignored. The radiation pattern for any parameter also can be obtained from those for monoclinic parameters using the chain rule in a similar way to equation 13 . To convert diffraction patterns to the reflection patterns from a horizontal reflector in an isotropic background (Oh and Alkhalifah, 2016a), incidence and diffraction angles are converted to the opening angle $\theta_{o}$, based on Snell's law as follows: 


$$
\theta_{o}^{\mathrm{P}-\mathrm{P}}=\theta_{d}^{\mathrm{P}}-\theta_{i}^{\mathrm{P}}
$$

where

$$
\theta_{d}^{\mathrm{P}}=-\theta_{i}^{\mathrm{P}}
$$

and

$$
\theta_{o}^{\mathrm{P}-\mathrm{SV}}=\theta_{d}^{\mathrm{SV}}-\theta_{i}^{\mathrm{P}}
$$

where

$$
\theta_{d}^{\mathrm{SV}}=\sin ^{-1}\left(\frac{v_{s}}{v_{p}} \sin \left(-\theta_{i}^{\mathrm{P}}\right)\right)
$$

\section{Parameterization I: Monoclinic parameterization}

The monoclinic parameterization has 13 independent parameters, $\tilde{C}_{I J}$, in equation 5 . Figure 2 shows the reflection patterns and horizontal slices of the gradient direction on the top of the L2 layer. In the radiation pattern, we observe that the influence of the $\tilde{C}_{I J}$ parameters is well-decoupled in the data. We observe four different types of parameters in terms of the azimuth direction; the narrow azimuth group $\left(\tilde{C}_{11}, \tilde{C}_{13}, \tilde{C}_{55}\right)$, the wide azimuth group $\left(\tilde{C}_{22}, \tilde{C}_{23}\right.$, $\left.\tilde{C}_{44}\right)$, the intermediate azimuth group $\left(\tilde{C}_{12}, \tilde{C}_{66}, \tilde{C}_{16}, \tilde{C}_{26}, \tilde{C}_{36}, \tilde{C}_{45}\right)$, and the azimuth independent parameter $\left(\tilde{C}_{33}\right)$. As a result, the single-shot gradient directions have a different coverage for each parameter. The opening angle in the radiation pattern is affected by the offset-to-depth ratio (Figure 1). Due to the limited offsetto-depth ratio (2:0.6 and approximately $120^{\circ}$ in terms of the opening angle) in this example, we observe a trade-off in the gradient direction for the groups $\left[\tilde{C}_{11}, \tilde{C}_{13}, \tilde{C}_{55}\right]$ and $\left[\tilde{C}_{22}, \tilde{C}_{23}, \tilde{C}_{44}\right]$. The strong

\section{PARAMETERIZATION STUDY}

In this section, we compare the behaviors of three possible parameterizations (Table 2), monoclinic, velocity-based, and hierarchical parameterizations based on the reflection patterns and the single-shot gradient direction. Figure 1 shows a schematic diagram illustrating a threelayer model, which has a water layer and a very weak ORT background. The strong azimuthally rotated ORT layer (L2) with rotation angle $\left(\phi=45^{\circ}\right)$ is interbedded. The initial model is the same as the background medium (water layer and L1), so that the residual wavefields only include reflection energy from the L2 layer. We assume single pressure source on the middle of the surface, and hydrophones are placed at all nodal points on the water surface. The maximum offset is $2 \mathrm{~km}$ along all azimuth directions. The gradient directions for each parameter can be calculated using equations 12 and 13. In Figure 1, the reflection patterns are calculated based on Snell's law assuming the reflection raypath from a source (red dot) to a receiver (green dot) via subsurface reflector on the top of L2 (yellow dot). The reflection patterns (Figures 2, 3, and 4) are displayed as a $2 \mathrm{D}$ plot in terms of the opening angle along reflection plane (blue line) with different azimuth angles (Oh and Alkhalifah, 2016a).
Table 2. The list of parameters in each parameterization.

$\begin{array}{lllllllllll}\text { Monoclinic } & \tilde{C}_{11} & \tilde{C}_{22} & \tilde{C}_{33} & \tilde{C}_{12} & \tilde{C}_{13} & \tilde{C}_{23} & \tilde{C}_{44} & \tilde{C}_{55} & \tilde{C}_{66} & - \\ & \tilde{C}_{16} & \tilde{C}_{26} & \tilde{C}_{36} & \tilde{C}_{45} & - & - & - & - & - & - \\ \text { Velocity-based } & v_{p 1} & v_{p 2} & v_{p 3} & v_{n 1} & v_{n 2} & v_{s 1} & v_{s 2} & v_{s 3} & \delta_{3} & \phi \\ \text { Hierarchical } & v_{p 1} & v_{s 1} & \varepsilon_{1} & \eta_{1} & \gamma_{1} & \varepsilon_{D} & \eta_{D} & \gamma_{D} & \delta_{3} & \phi\end{array}$

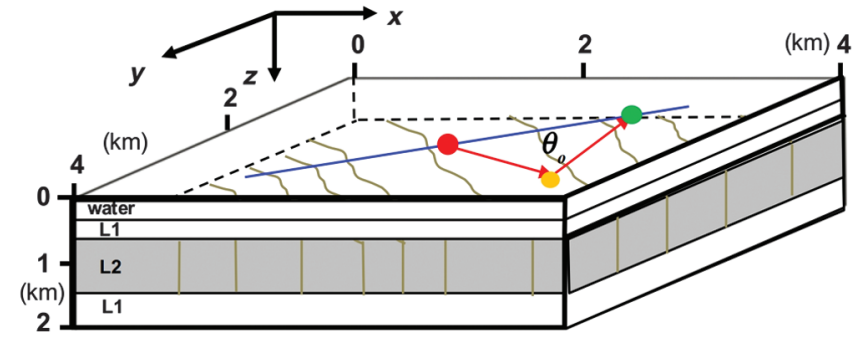

Figure 1. The geometry of a three-layer model. The model has a very weak ORT background, so that we can ignore anisotropic propagation effects in the radiation-pattern analysis. The model also has strong azimuthally rotated ORT layer, L2 $\left(\phi=45^{\circ}\right)$. The red, green, and yellow dots denote the possible source, receiver, and perturbation points for reflection data, respectively. The red arrows indicate the reflection raypath from the top of anomalous layer (L2), which are assumed to derive the reflection pattern from Figures 2-4.

Table 1. The moment-tensor forms of the virtual source for $\mathbf{1 3}$ monoclinic parameters.

$$
\begin{aligned}
& \mathbf{M}_{\tilde{C}_{11}} \\
& \mathbf{M}_{\tilde{C}_{22}} \\
& \mathbf{M}_{\tilde{C}_{33}} \\
& \mathbf{M}_{\tilde{C}_{12}} \\
& \mathbf{M}_{\tilde{C}_{13}} \\
& \mathbf{M}_{\tilde{C}_{23}} \\
& \mathbf{M}_{\tilde{C}_{66}} \\
& \left(\begin{array}{ccc}
\varepsilon_{x} & 0 & 0 \\
0 & 0 & 0 \\
0 & 0 & 0
\end{array}\right) \\
& \left(\begin{array}{ccc}
0 & 0 & 0 \\
0 & \boldsymbol{\varepsilon}_{y} & 0 \\
0 & 0 & 0
\end{array}\right) \\
& \left(\begin{array}{ccc}
0 & 0 & 0 \\
0 & 0 & 0 \\
0 & 0 & \varepsilon_{z}
\end{array}\right) \\
& \left(\begin{array}{ccc}
\boldsymbol{\varepsilon}_{y} & 0 & 0 \\
0 & \boldsymbol{\varepsilon}_{x} & 0 \\
0 & 0 & 0
\end{array}\right) \\
& \left(\begin{array}{ccc}
\varepsilon_{z} & 0 & 0 \\
0 & 0 & 0 \\
0 & 0 & \varepsilon_{x}
\end{array}\right) \\
& \left(\begin{array}{ccc}
0 & 0 & 0 \\
0 & \boldsymbol{\varepsilon}_{z} & 0 \\
0 & 0 & \boldsymbol{\varepsilon}_{y}
\end{array}\right) \\
& 2\left(\begin{array}{ccc}
0 & \boldsymbol{\varepsilon}_{x y} & 0 \\
\boldsymbol{\varepsilon}_{x y} & 0 & 0 \\
0 & 0 & 0
\end{array}\right) \\
& \mathbf{M}_{\tilde{C}_{44}} \\
& \mathbf{M}_{\tilde{C}_{55}} \\
& \mathbf{M}_{\tilde{C}_{16}} \\
& \mathbf{M}_{\tilde{C}_{26}} \\
& \mathbf{M}_{\tilde{C}_{36}} \\
& \mathbf{M}_{\tilde{C}_{45}} \\
& 2\left(\begin{array}{ccc}
0 & 0 & 0 \\
0 & 0 & \boldsymbol{\varepsilon}_{y z} \\
0 & \boldsymbol{\varepsilon}_{y z} & 0
\end{array}\right) 2\left(\begin{array}{ccc}
0 & 0 & \boldsymbol{\varepsilon}_{x z} \\
0 & 0 & 0 \\
\boldsymbol{\varepsilon}_{x z} & 0 & 0
\end{array}\right)\left(\begin{array}{ccc}
2 \boldsymbol{\varepsilon}_{x y} & \boldsymbol{\varepsilon}_{x x} & 0 \\
\boldsymbol{\varepsilon}_{x x} & 0 & 0 \\
0 & 0 & 0
\end{array}\right)\left(\begin{array}{ccc}
0 & \boldsymbol{\varepsilon}_{y y} & 0 \\
\boldsymbol{\varepsilon}_{y y} & 2 \boldsymbol{\varepsilon}_{x y} & 0 \\
0 & 0 & 0
\end{array}\right)\left(\begin{array}{ccc}
0 & \boldsymbol{\varepsilon}_{y y} & 0 \\
\boldsymbol{\varepsilon}_{y y} & 0 & 0 \\
0 & 0 & 2 \boldsymbol{\varepsilon}_{x y}
\end{array}\right) 2\left(\begin{array}{ccc}
0 & 0 & \boldsymbol{\varepsilon}_{y z} \\
0 & 0 & \boldsymbol{\varepsilon}_{x z} \\
\boldsymbol{\varepsilon}_{y z} & \boldsymbol{\varepsilon}_{x z} & 0
\end{array}\right)
\end{aligned}
$$


coupling in the groups $\left[\tilde{C}_{12}, \tilde{C}_{66}\right]$ and $\left[\tilde{C}_{36}, \tilde{C}_{45}\right]$ is also observed. Although the radiation patterns show some overlap in the group $\left[\tilde{C}_{16}, \tilde{C}_{26}, \tilde{C}_{12}, \tilde{C}_{66}\right]$, the trade-off shown in the gradient direction is not strong thanks to the polarity change along the azimuth direction. As a result, the additional parameters $\left(\tilde{C}_{16}, \tilde{C}_{26}, \tilde{C}_{36}, \tilde{C}_{45}\right)$ might be inverted well with less trade-off, as Rusmanugroho et al. (2017) also show for 2D elastic tilted transverse isotropic FWI. However, the monoclinic FWI is not necessary when we consider fracture-induced anisotropy, where vertically aligned fractures are azimuthally rotated. As the Bond transformation shows in equation 8 , the number of independent parameters reduces from 13 to 10 under the assumption for the azimuthally rotated ORT medium.

\section{Parameterization II: Velocity-based parameterization}

Under the rotated ORT assumption, we can parameterize the ORT $C_{I J}$ parameters in several ways (Oh and Alkhalifah, 2016a). One common strategy defines a medium with $\mathrm{P}-\left(v_{p}\right), \mathrm{S}-\left(v_{s}\right)$, and NMO velocities $\left(v_{n}\right)$ as follows:

$$
\left(\begin{array}{llllllllll}
v_{p 3} & v_{p 1} & v_{p 2} & v_{n 1} & v_{n 2} & \delta_{3} & v_{s 1} & v_{s 2} & v_{s 3} & \phi
\end{array}\right) .
$$

The parameters $v_{p 1}, v_{p 2}$, and $v_{p 3}$ denote the P-wave velocities in the $x$-, $y$-, and $z$-directions, respectively. The parameters $v_{n 1}$ and $v_{n 2}$ are the NMO velocities for horizontal reflectors in the $x z$ - and $y z$-planes, respectively. The parameters $v_{s 1}$ and $v_{s 2}$ indicate the vertical S-wave velocities that are polarized in the $x z$ - and $y z$-planes, respectively, and $v_{s 3}$ is the horizontal S-wave velocity in the $x z$-plane, but propagating in the $x$-direction. The parameter $\delta_{3}$ is an anisotropic parameter in the $x y$-plane (Thomsen, 1986; Tsvankin, 1997). The ORT $C_{I J}$ parameters are expressed using the velocity-based parameterization as follows:

$$
\left(\begin{array}{l}
C_{11} \\
C_{22} \\
C_{33} \\
C_{12} \\
C_{13} \\
C_{23} \\
C_{44} \\
C_{55} \\
C_{66}
\end{array}\right)=\left(\begin{array}{c}
\rho v_{p 1}^{2} \\
\rho v_{p 2}^{2} \\
\rho v_{p 3}^{2} \\
\rho\left[\sqrt{\left(v_{p 1}^{2}-v_{s 3}^{2}\right)\left(\left(1+2 \delta_{3}\right) v_{p 1}^{2}-v_{s 3}^{2}\right)}-v_{s 3}^{2}\right] \\
\rho\left[\sqrt{\left(v_{p 3}^{2}-v_{s 1}^{2}\right)\left(v_{n 1}^{2}-v_{s 1}^{2}\right)}-v_{s 1}^{2}\right] \\
\rho\left[\sqrt{\left(v_{p 3}^{2}-v_{s 2}^{2}\right)\left(v_{n 2}^{2}-v_{s 2}^{2}\right)}-v_{s 2}^{2}\right] \\
\rho v_{s 2}^{2} \\
\rho v_{s 1}^{2} \\
\rho v_{s 3}^{2}
\end{array}\right) .
$$

\section{Monoclinic parameterization}
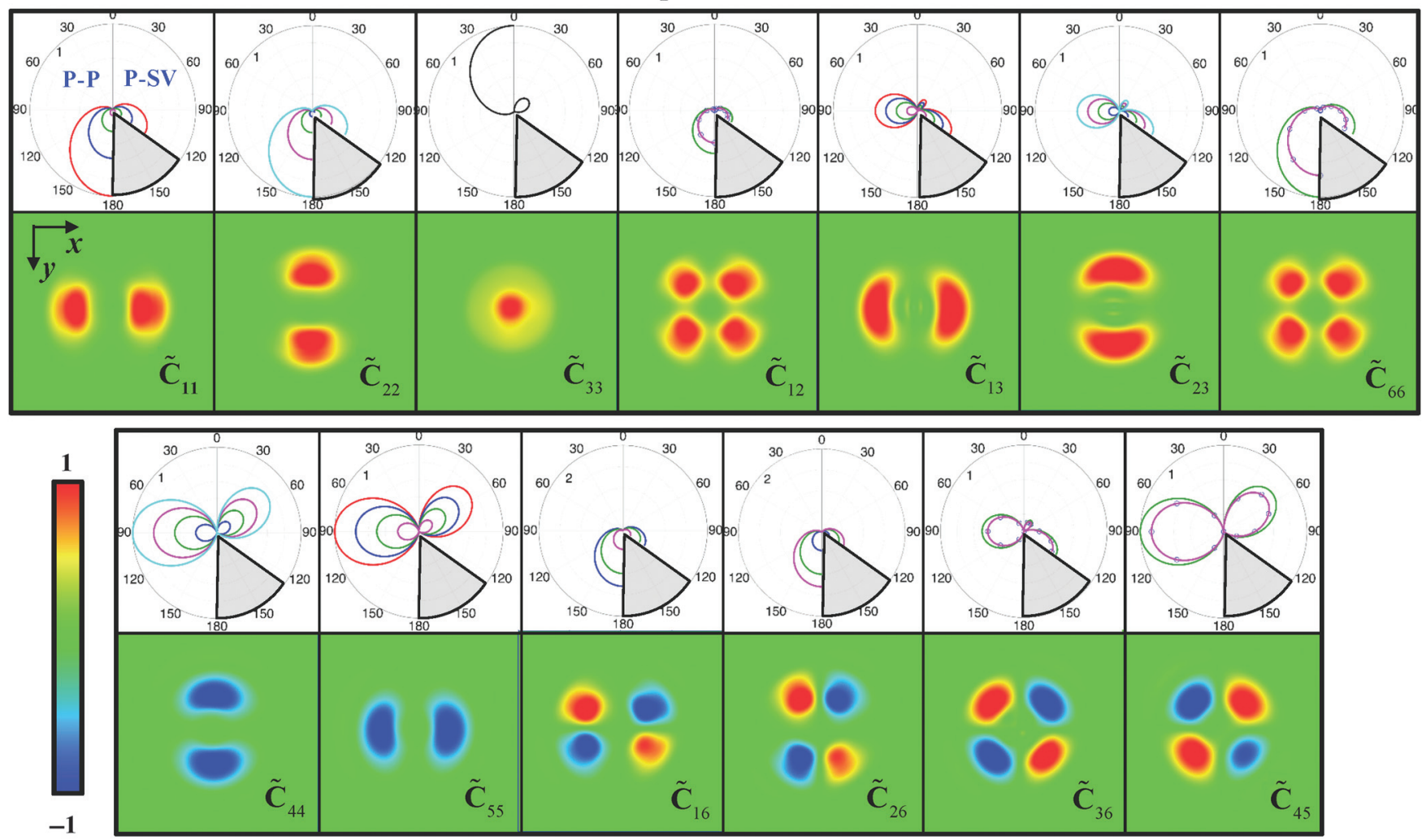

Azimuth $\left(\varphi_{\mathrm{sr}}\right):=0^{\circ}-30^{\circ}-45^{\circ}-60^{\circ}-90^{\circ}-$ All

Figure 2. The first row shows the P-P (left hemisphere) and P-SV (right hemisphere) radiation pattern for 13 monoclinic elastic constants. The angle is defined as an opening angle satisfying Snell's law from a horizontal reflector. The Poisson's ratio is assumed to be 0.25 to calculate $\mathrm{P}-\mathrm{SV}$ radiation pattern $\left(\mathrm{Oh}\right.$ and Alkhalifah, 2016a). Notice that $\varphi_{\mathrm{sr}}$ is the azimuth angle of a source-receiver line in Figure 1, which is not related to the rotation angle of the symmetric axis $\varphi$. The second row shows the horizontal slices of gradient directions for each parameter on the top of anomaly (L2) acquired by a single source (red dot in Figure 1). Note that background has very weak ORT anisotropy to ignore propagation effects in anisotropic media. 
The attractive feature of the velocity-based parameterization is that each parameter has the same units (except $\boldsymbol{\delta}_{3}$ ), so that we can easily scale the gradient direction. However, the velocity-based parameterization has complex behavior during FWI as we observed in the radiation patterns (Oh and Alkhalifah, 2016a). Figure 3 shows the P-P and P-SV radiation patterns of the velocity-based parameterization. At first, we observe outstanding separation of $\left[v_{p 1}, v_{n 1}, v_{s 1}\right]$ and $\left[\mathrm{v}_{p 2}, v_{n 2}, v_{s 2}\right]$ along the azimuth direction, which means that the trade-off between each pair of parameters is not strong. However, this also means that the FWI updates would always be ORT if we do not apply some constraints on the parameter updates. This ORT update causes complicated wave propagation in the inversion model. For this reason, suitable constraints on the updates should be applied to stabilize the FWI procedure at an early stage.

Regarding the rotation of ORT axes, we observe that $v_{p 3}$ has azimuth independent radiation pattern, whereas other parameters depend on the rotation angle of the reflection plane. The sensitivity of $v_{s 3}$ is the weakest. Supporting these observations from radiation-pattern plots, the single-shot gradient direction (Figure 5) also shows azimuth-dependent characteristics of the parameter perturbations in the velocity-based parameterization. The first row is the horizontal slices of the gradient direction on the top of the anomaly when the initial rotation angle is $0^{\circ}$, and the second row shows those obtained when the initial rotation angle is $30^{\circ}$. Considering that the true rotation angle is $45^{\circ}$, the gradients of each parameter will match the wavefield along the wrong azimuth direction when the initial rotation angle is far from the true angle. This means that with the velocity-based parameterization, we need a good enough ORT background and a good initial estimation for the rotation angle.

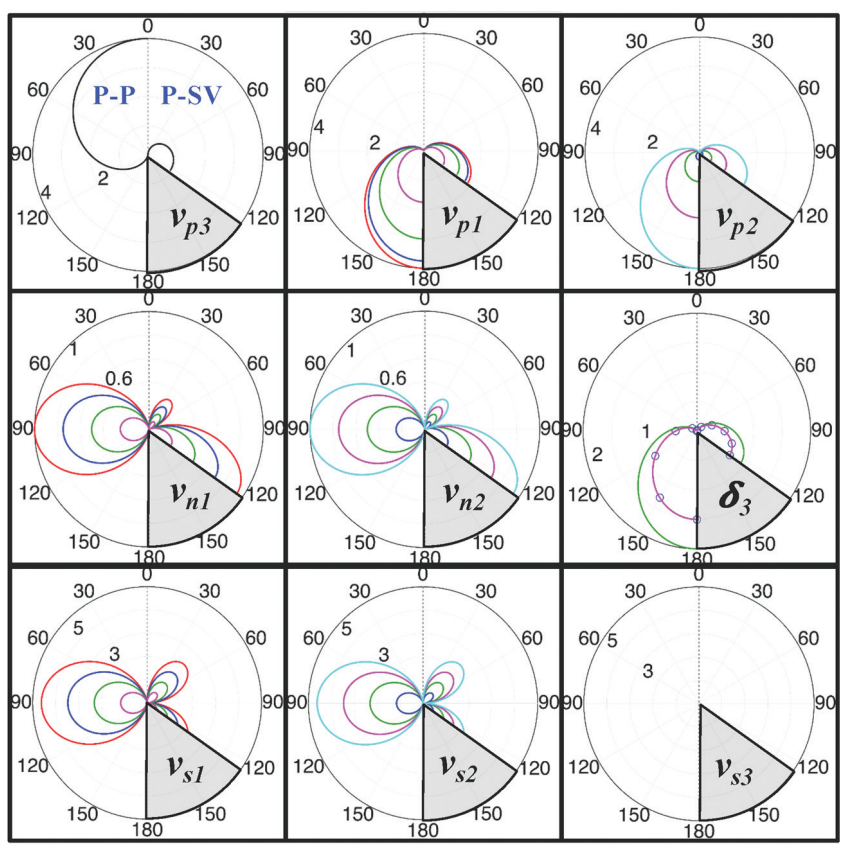

Azimuth $\left(\varphi_{\mathrm{sr}}\right):-0^{\circ}-30^{\circ}-45^{\circ}-60^{\circ}-90^{\circ}-$ All

Figure 3. The same as Figure 2, but for the velocity-based parameterization when the rotation angle is $0^{\circ}(\mathrm{Oh}$ and Alkhalifah, 2016a).

\section{Parameterization III: Hierarchical parameterization}

To avoid the aforementioned complexities in multiparameter FWI for rotated ORT media, the hierarchical parameterization has been proposed with three deviation parameters as follows (Oh and Alkhalifah, 2016a):

$$
\left(\begin{array}{llllllllll}
v_{p 1} & v_{s 1} & \varepsilon_{1} & \eta_{1} & \gamma_{1} & \delta_{3} & \varepsilon_{D} & \eta_{D} & \gamma_{D} & \phi
\end{array}\right)
$$

where

$$
\begin{gathered}
\varepsilon_{D}=\frac{\varepsilon_{2}-\varepsilon_{1}}{1+2 \varepsilon_{1}} \\
\eta_{D}=\frac{\eta_{2}-\eta_{1}}{1+2 \eta_{1}},
\end{gathered}
$$

and

$$
\gamma_{D}=\frac{\gamma_{1}-\gamma_{2}}{1+2 \gamma_{2}}
$$

The parameters $\left[\varepsilon_{1}, \gamma_{2}\right]$ and $\left[\varepsilon_{2}, \gamma_{1}\right]$ denote the VTI parameters $\varepsilon$ and $\gamma$ in the $x z$ - and $y z$-planes, respectively. Instead of VTI parameter $\boldsymbol{\delta}_{1}$, we use $\eta_{1}\left(v_{n 1}=v_{p 3} \sqrt{1+2 \delta_{1}}\right.$ and $\left.v_{p 1}=v_{n 1} \sqrt{1+2 \eta_{1}}\right)$ suggested by Alkhalifah (2003). In this VTI combination, the influence of parameter $\eta$ is negligible (Alkhalifah, 2016). The deviation parameters $\left(\varepsilon_{D}, \eta_{D}\right.$, and $\left.\gamma_{D}\right)$ determine azimuthal variations of each anisotropic parameter on the $x y$-plane (Tsvankin, 1997; Masmoudi and Alkhalifah, 2016). The ORT $C_{I J}$ parameters are expressed by the hierarchical parameterization as follows:

$$
\left(\begin{array}{c}
C_{11} \\
C_{22} \\
C_{33} \\
C_{12} \\
C_{13} \\
C_{23} \\
C_{44} \\
C_{55} \\
C_{66}
\end{array}\right)=\left(\begin{array}{c}
\rho v_{p 1}^{2} \\
\left(1+2 \varepsilon_{D}\right) \rho v_{p 1}^{2} \\
\frac{\rho v_{p 1}^{2}}{\left(1+2 \varepsilon_{1}\right)} \\
\rho\left[\sqrt{\left(v_{p 1}^{2}-\left(1+2 \gamma_{1}\right) v_{s 1}^{2}\right)\left(\left(1+2 \delta_{3}\right) v_{p 1}^{2}-\left(1+2 \gamma_{1}\right) v_{s 1}^{2}\right)}-\left(1+2 \gamma_{1}\right) v_{s 1}^{2}\right] \\
\rho\left[\sqrt{\left(\frac{v_{p 1}^{2}}{\left(1+2 \varepsilon_{1}\right)}-v_{s 1}^{2}\right)\left(\frac{v_{p 1}^{2}}{\left(1+2 \eta_{1}\right)}-v_{s 1}^{2}\right)}-v_{s 1}^{2}\right] \\
\rho\left[\sqrt{\left(\frac{v_{p 1}^{2}}{\left(1+2 \varepsilon_{1}\right)}-\left(1+2 \gamma_{D}\right) v_{s 1}^{2}\right)\left(\frac{\left(1+2 \varepsilon_{D}\right) v_{p 1}^{2}}{\left(1+2 \eta_{1}\right)\left(1+2 \eta_{D}\right)}-\left(1+2 \gamma_{D}\right) v_{s 1}^{2}\right)}-\left(1+2 \gamma_{D}\right) v_{s 1}^{2}\right] \\
\left(1+2 \gamma_{D}\right) v_{s 1}^{2} \\
\left.\rho v_{s 1}^{2}\right) \\
\left(1+2 \gamma_{1}\right) v_{s 1}^{2}
\end{array}\right) .
$$

As Oh and Alkhalifah (2016a) show from the radiation-pattern analysis, this parameterization enables us to build up subsurface models from isotropic $\left(v_{p 1}\right.$ and $\left.v_{s 1}\right)$ to VTI $\left(\varepsilon_{1}, \eta_{1}\right.$, and $\left.\gamma_{1}\right)$ and finally to ORT media $\left(\varepsilon_{D}, \eta_{D}, \gamma_{D}\right.$, and $\left.\delta_{3}\right)$ by sequentially updating those parameters.

For rotated ORT media, this parameterization also offers better functionality compared with the velocity-based parameterization. In the reflection pattern (Figure 4), we observed that all five parameters $\left(v_{p 1}, v_{s 1}, \varepsilon_{1}, \eta_{1}\right.$, and $\left.\gamma_{1}\right)$ have azimuth-independent radiation patterns. In Figure 5, the single-shot gradient directions of these parameters are not sensitive to background rotation angle, which supports that we can recover the VTI model regardless of the rotation angle of ORT anisotropy. From this VTI model, we can further build a subsurface ORT model by updating deviation parameters $\left(\varepsilon_{D}, \eta_{D}\right.$, 
$\gamma_{D}$, and $\boldsymbol{\delta}_{3}$ ). However, at this stage, the estimation of deviation parameters is affected by the initial guess of the rotation angle.

\section{MULTISTAGE FWI IN ROTATED ORT MEDIA}

To enhance the convergence of multiparameter FWI, an accurate rotation angle is required. First, we examine the possibility of inverting for the rotation angle as an FWI target by analyzing the gradient direction and radiation patterns, as we have done for ORT parameters. Then, we suggest an optimal FWI update strategy, in which we update the subsurface model from simple to complex anisotropy using the hierarchical parameterization.

\section{Rotation angle as a FWI target}

The gradient direction of the rotation angle can be derived using the chain rule in equation 13 as follows:

$$
\begin{aligned}
& \nabla_{\phi} E=\partial \tilde{C}_{11, \phi} \nabla_{\tilde{C}_{11}} E+\partial \tilde{C}_{22, \phi} \nabla_{\tilde{C}_{22}} E+\partial \tilde{C}_{12, \phi} \nabla_{\tilde{C}_{12}} E \\
& \quad+\partial \tilde{C}_{13, \phi} \nabla_{\tilde{C}_{13}} E+\partial \tilde{C}_{23, \phi} \nabla_{\tilde{C}_{23}} E \\
& \quad+\partial \tilde{C}_{44, \phi} \nabla_{\tilde{C}_{44}} E+\partial \tilde{C}_{55, \phi} \nabla_{\tilde{C}_{55}} E+\partial \tilde{C}_{66, \phi} \nabla_{\tilde{C}_{66}} E \\
& \quad+\partial \tilde{C}_{16, \phi} \nabla_{\tilde{C}_{16}} E+\partial \tilde{C}_{26, \phi} \nabla_{\tilde{C}_{26}} E+\partial \tilde{C}_{36, \phi} \nabla_{\tilde{C}_{36}} E \\
& \quad+\partial \tilde{C}_{45, \phi} \nabla_{\tilde{C}_{45}} E
\end{aligned}
$$

where

$$
\left(\begin{array}{c}
\partial \tilde{C}_{11, \phi} \\
\partial \tilde{C}_{22, \phi} \\
\partial \tilde{C}_{33, \phi} \\
\partial \tilde{C}_{12, \phi} \\
\partial \tilde{C}_{13, \phi} \\
\partial \tilde{C}_{23, \phi} \\
\partial \tilde{C}_{44, \phi} \\
\partial \tilde{C}_{55, \phi} \\
\partial \tilde{C}_{66, \phi} \\
\partial \tilde{C}_{16, \phi} \\
\partial \tilde{C}_{26, \phi} \\
\partial \tilde{C}_{36, \phi} \\
\partial \tilde{C}_{45, \phi}
\end{array}\right)=\left(\begin{array}{c}
4 \sin \phi \cos \phi\left\{\left(-C_{11}+C_{12}+2 C_{66}\right) \cos ^{2} \phi-\left(-C_{22}+C_{12}+2 C_{66}\right) \sin ^{2} \phi\right\} \\
4 \sin \phi \cos \phi\left\{\left(-C_{22}+C_{12}+2 C_{66}\right) \cos ^{2} \phi-\left(-C_{11}+C_{12}+2 C_{66}\right) \sin ^{2} \phi\right\} \\
0 \\
2\left(C_{11}+C_{22}-2 C_{12}-4 C_{66}\right) \sin \phi \cos \phi\left(\cos ^{2} \phi-\sin ^{2} \phi\right) \\
2\left(C_{23}-C_{13}\right) \sin \phi \cos \phi \\
-2\left(C_{23}-C_{13}\right) \sin \phi \cos \phi \\
2\left(C_{55}-C_{44}\right) \sin \phi \cos \phi \\
-2\left(C_{55}-C_{44}\right) \sin \phi \cos \phi \\
\left(-C_{11}+C_{12}+2 C_{66}\right)\left(\cos ^{4} \phi-3 \sin ^{2} \phi \cos ^{2} \phi\right)+\left(-C_{22}+C_{12}+2 C_{66}\right)\left(\sin ^{4} \phi-3 \sin ^{2} \phi \cos ^{2} \phi\right) \\
\left(C_{22}-C_{12}-2 C_{66}\right)\left(\cos ^{4} \phi-3 \sin ^{2} \phi \cos ^{2} \phi\right)+\left(C_{11}-C_{12}-2 C_{66}\right)\left(\sin ^{4} \phi-3 \sin ^{2} \phi \cos ^{2} \phi\right) \\
\left(C_{23}-C_{13}\right)\left(\cos ^{2} \phi-\sin ^{2} \phi\right) \\
\left(C_{44}-C_{55}\right)\left(\cos ^{2} \phi-\sin ^{2} \phi\right)
\end{array}\right)
$$

As shown in equation 30, gradient direction of the rotation angle depends on 12 monoclinic parameters except $\tilde{C}_{33}$, which causes complicated behavior of the rotation angle as an FWI target. Here, we show some interesting features in the gradient direction of the rotation angle.

At first, the gradient direction of the rotation angle depends on the model parameter deviation along slow and fast axes of ORT media such as $\left(C_{23}-C_{13}\right),\left(C_{55}-C_{44}\right)$, and $\left(C_{11}+C_{22}-2 C_{12}-4 C_{66}\right)$. In other words, the activation of each monoclinic gradient is determined by the properties of the initial model (or the inverted model during FWI). For example, if the inversion model is isotropic and VTI, the gradient direction of the rotation angle is zero because all the coefficients in equation 31 are zero. This behavior is also physically reasonable because isotropic and VTI models are azimuthally invariant. However, once we have ORT background model, the rotation angle can be estimated through FWI procedure. Figure 6 shows the radiation pattern and single-shot gradient direction of the rotation angle. Here, four different triggers (type I, $C_{22}>C_{11}$; type II, $C_{23}>C_{13}$; type III, $C_{44}>C_{55}$; and type IV, $C_{12}>C_{66}$ ), which affect the radiation patterns of the rotation angle in different ways, are displayed. We expect that, depending on the model parameter deviations in the inversion model, the sensitivity of the rotation angle can be changed abruptly during FWI.

Second, we also observe polarity change in the gradient direction of the rotation angle along the azimuth direction as is also observed from the eikonal equation in the previous literature (Masmoudi et al., 2017). This azimuthal polarity changes in the gradient direction (or sensitivity kernel) indicates that the gradient direction of the rotation angle is measured by the azimuthal variation of the wavefields. From this observation, we expect that the trade-off between the rotation angle and ORT parameters are not strong although the amplitude variations in the radiation pattern of the rotation angle resemble some ORT parameters in Figures 3 and 4.

\section{Multistage FWI strategy}

Based on above observations on the rotation angle as an FWI target, we suggest the optimal multistage FWI strategy using the hierarchical parameterization because the hierarchical parameterization shows better behavior than the velocity-based parameterization. As mentioned in the parameterization study, most parameters in the velocity-based parameterization are sensitive to the background rotation angle, which means that accurate rotation angle information is required. In addition, if we invert for all nine ORT parameters simultaneously using the velocity-based parameterization, inversion

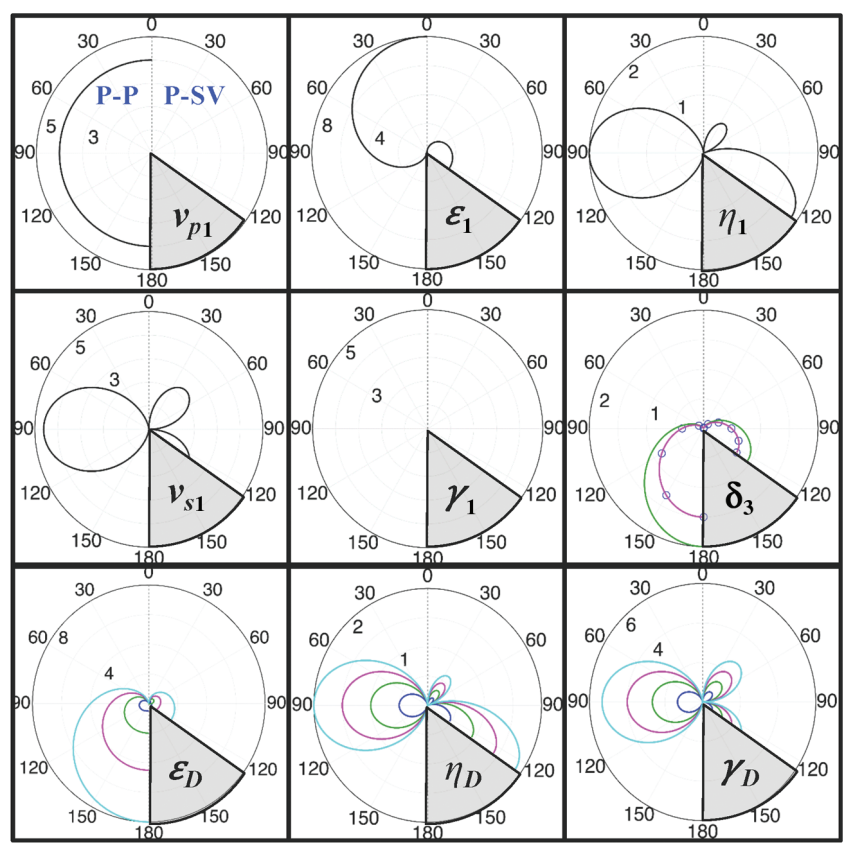

Azimuth $\left(\varphi_{\mathrm{sr}}\right):=0^{\circ}-30^{\circ}-45^{\circ}-60^{\circ}-90^{\circ}-$ All

Figure 4. The same as Figure 2, but for the hierarchical parameterization when the rotation angle is $0^{\circ}$ (Oh and Alkhalifah, 2016a). 
model is ORT from the beginning of FWI. Therefore, the complexity of FWI for the rotation angle increases combining all four types in Figure 6. To prevent this complexity in FWI for the rotation angle, a proper constraint needs to be applied.

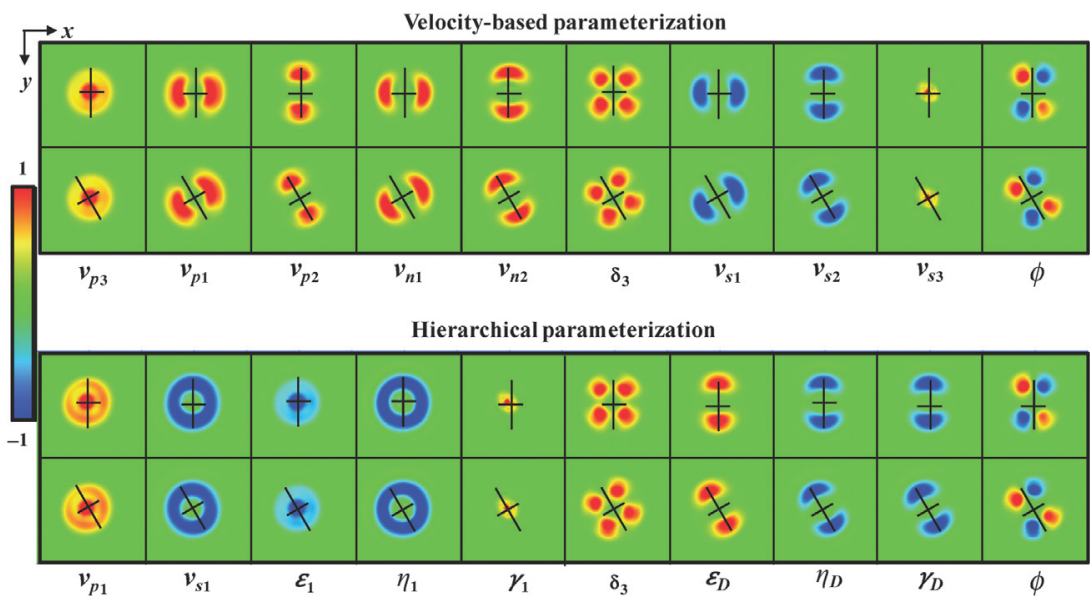

Figure 5. The single-shot gradient directions on the top of the anomaly for two different parameterizations when rotation angles $\phi$ in FWI model are $0^{\circ}$ and $30^{\circ}$, respectively. The long and short black lines indicate fast and slow axes of initial ORT background.

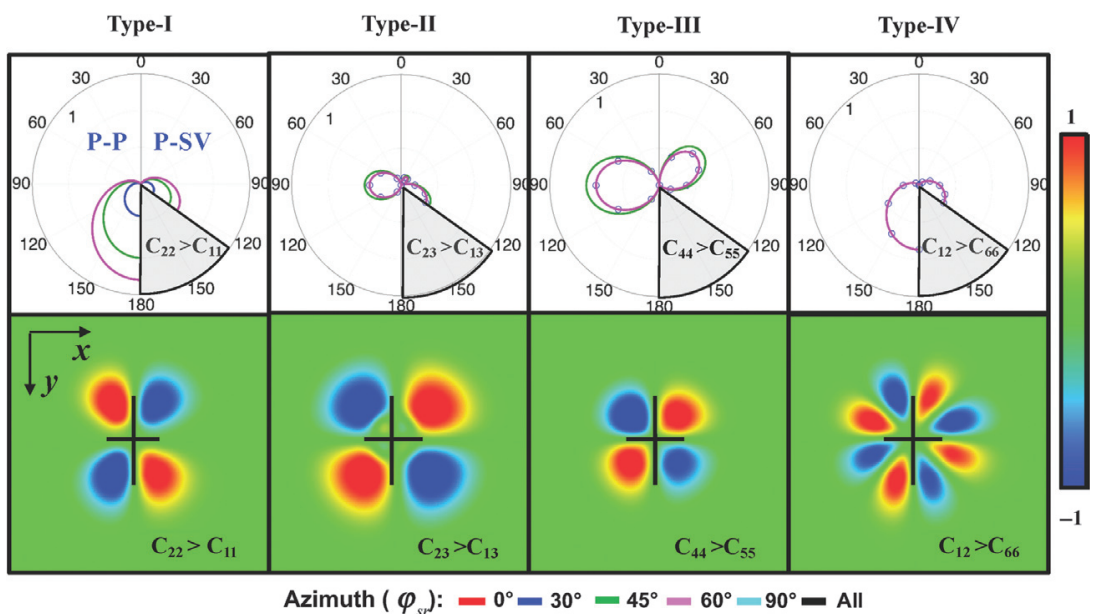

Figure 6. The radiation patterns and the single-shot gradient directions for the azimuth angle $\phi$ perturbation. Note that the behavior of the azimuth angle depends on different model parameter deviations in the background media, as shown in equations 30 and 31. a)

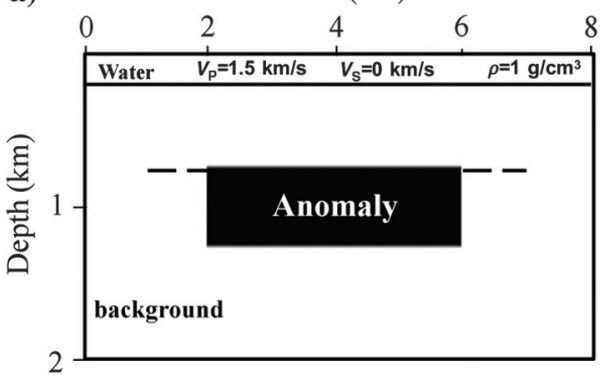

b)

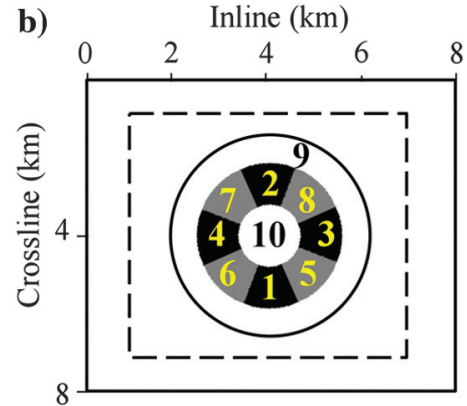

Figure 7. (a) The vertical and (b) horizontal slices of the hockey-puck model. The model has an anomalous layer, which has hockey-puck-shaped parameter perturbations horizontally. The parameter perturbations are listed in Table 3.
On the other hand, five parameters $\left(v_{p 1}, v_{s 1}, \varepsilon_{1}, \eta_{1}\right.$, and $\left.\gamma_{1}\right)$ in the hierarchical parameterization are robust to azimuthal variation of the wavefields, which means that we can build a VTI model regardless of true rotation angle. In addition, using the hierarchical parameterization, we can easily constrain the subsurface model by freezing some anisotropic parameters locally or globally. For example, if we invert for only $\varepsilon_{D}$ and freeze other deviation parameters $\left(\eta_{D}, \gamma_{D}\right.$, and $\left.\boldsymbol{\delta}_{3}\right)$, the sensitivity of the rotation angle is governed by only types-I and -II, as expected from equation 29, whose amplitude variations of the radiation pattern resemble those from $\varepsilon_{D}$ perturbation in Figure 4. This constraint on the ORT update allows us to recover the rotation angle with wide opening angle components of the data, which are also used to invert for $\varepsilon_{D}$. However, because the rotation angle is estimated by measuring azimuthal variations of the wavefields as observed in Figure 6, the trade-off between $\varepsilon_{D}$ and the rotation angle is not strong.

As Oh and Alkhalifah (2016a) show, four parameters $\left(v_{s 1}, \eta_{1}, \eta_{D}\right.$, and $\left.\gamma_{D}\right)$ are strongly coupled and two parameters $\varepsilon_{D}$ and $\boldsymbol{\delta}_{3}$ are also coupled. In addition, $\gamma_{1}$ is not sensitive to the P-P and P-SV modes. For this reason, inverting for only five parameters $\left(v_{p 1}, v_{s 1}, \varepsilon_{1}, \varepsilon_{D}\right.$, and $\left.\phi\right)$ is a reasonable choice for a marine data inversion. Using these five parameters, we first build an isotropic background model by inverting for only $v_{p 1}$ and $v_{s 1}$. After the objective function reaches a certain criterion, we add $\varepsilon_{1}$ in the inversion process to build VTI model. Then, the deviation parameter $\varepsilon_{D}$ is added to recover the azimuthal variation. The parameters $\varepsilon_{1}$ and $\varepsilon_{D}$ can be added together to reduce the number stages because they are welldecoupled in the data, as supported by the radiation patterns (Figure 4). Finally, we add the rotation angle in the FWI procedure to fix the error in $\varepsilon_{D}$ and to find the true rotation angle.

\section{TRADE-OFF ANALYSIS}

To prove the trade-off patterns observed in the radiation-pattern analysis, we conduct trade-off analysis using a simple synthetic model. To design a synthetic model that reveals the trade-off between parameters, we allocate each model parameter perturbation a unique location in the model to observe imprints from other parameters (Köhn et al., 2012, 2015). To do this, we design a simple layered model with a disc-shaped anomaly, which we refer to as the hockey puck model, as shown in Figure 7.

The first layer is water, and the background ORT parameters are listed in Table 3 . To verify the trade-off between parameters, each ORT parameter perturbation is located at a different part of the hockey puck from areas 1 to 9 . The true rotation angle perturbation occurs at only the inner part (area 10). The detailed parameter per- 
turbation amount for each parameterization is also listed in Table 3. A total of 400 pressure sources are uniformly placed on the water surface with a $400 \mathrm{~m}$ interval along the inline and crossline directions. For receivers, 160,000 hydrophones are installed at every nodal point on the water surface. For each shot, the maximum offset is limited to $4 \mathrm{~km}$ along all azimuth directions by deactivating the receivers outside $4 \mathrm{~km}$ offset limit. This offset control is needed to maintain a fixed-offset range for all the parameter perturbations. The initial model is the same as the background model, so that the only difference is caused by the anomaly. Considering the target depth $(800 \mathrm{~m})$, the offset-to-depth ratio is $5: 1$, which means that the maximum opening angle for a reflection from an anomaly is approximately $136^{\circ}$ in this example. For this hockey-puck model, we show the gradient directions of each parameter in the velocity-based and hierarchical parameterizations depending on different initial rotation angles $\left(0^{\circ}, 45^{\circ}\right.$, and $\left.90^{\circ}\right)$ on the whole puck (covering areas $1-10)$.

Figures 8 and 9 show the horizontal slices of the gradient directions on top of the anomaly (dashed black line in Figure 7a) depending on the different initial rotation angle for the velocity-based and the hierarchical parameterization, respectively. For visualization, the area within the dashed black line (Figure 7b) is displayed. At the end of each row, red and blue lines indicate the true fast axes of the background ORT medium in the center of the puck (area 10) and the rest of the puck (from areas 1 to 9), respectively. The ellipsoid denotes the initial guess for the ORT axis in the whole puck (from areas 1 to 10). In Figure 7, note that the true rotation angles in the rest of the puck (areas 1-9) and the inner part (area 10), are $0^{\circ}$ and $45^{\circ}$, respectively. In each slice, the black arrow indicates the true location of each parameter. The imprints in other locations result from the trade-off between parameters.

In the first row of Figure 8, the velocity-based parameterization shows a nice separation of the gradient direction, as we observed in the single-shot gradient direction in Figure 5. The parameter $v_{p 3}$ is well-isolated (slice E0). Two horizontal P-wave velocities $\left(v_{p 1}\right.$ and $\left.v_{p 2}\right)$ are also well-decoupled, although the trade-off with $\boldsymbol{\delta}_{3}$ is observed (slices E1 and E2). Two NMO velocities $\left(v_{n 1}\right.$ and $\left.v_{n 2}\right)$ are barely detected due to the trade-off between the P- and S-wave velocities (slices E3 and E4). The anisotropic parameter $\boldsymbol{\delta}_{3}$ is wellisolated (slice E5). Two vertical S-wave velocities $\left(v_{s 1}\right.$ and $\left.v_{s 2}\right)$ suffer from the trade-off with the P-wave velocities (slices E6 and E7). The parameter $v_{s 3}$ (slice E8) is not recoverable, whereas the gradient direction of the rotation angle (slice E9) is well-detected. Because we only record $\mathrm{P}$-waves using hydrophones, the S-wave velocities are generally not recovered. In addition, in these PP conversions, the $\mathrm{S}$-wave velocities strongly suffer from trade-off with P-wave velocities. In the second and third rows of Figure 8, the initial guess for the rotation angle in the outer part is $45^{\circ}$ and $90^{\circ}$

Table 3. The background model parameters for the hockey-puck model in Figure 7 and perturbed values for each parameterization. Note that the perturbed values in each parameterization are determined to provide a similar influence on the data.

\begin{tabular}{ccccccccccc} 
Initial (background) & $v_{p 1}$ & $v_{s 1}$ & $\varepsilon_{1}$ & $\varepsilon_{2}$ & $\eta_{1}$ & $\eta_{2}$ & $\delta_{3}$ & $\gamma_{1}$ & $\gamma_{2}$ & $\Phi(1-10)$ \\
& $2 \mathrm{~km} / \mathrm{s}$ & $1.15 \mathrm{~km} / \mathrm{s}$ & 0 & 0.045 & 0 & 0.045 & 0 & 0.045 & 0 & $0 \% / 45^{\circ} / 90^{\circ}$ \\
Parameterization I & $1 . d v_{p 3}$ & $2 . d v_{s 1}$ & $3 . d v_{p 2}$ & $4 . d v_{p 1}$ & $5 . d v_{n 2}$ & $6 . d v_{n 1}$ & $7 . d \delta_{3}$ & $8 . d v_{s 2}$ & $9 . d v_{s 1}$ & $10 . d \Phi$ \\
& $0.2 \mathrm{~km} / \mathrm{s}$ & $0.115 \mathrm{~km} / \mathrm{s}$ & $0.2 \mathrm{~km} / \mathrm{s}$ & $0.2 \mathrm{~km} / \mathrm{s}$ & $0.2 \mathrm{~km} / \mathrm{s}$ & $0.2 \mathrm{~km} / \mathrm{s}$ & 0.105 & $0.115 \mathrm{~km} / \mathrm{s}$ & $0.115 \mathrm{~km} / \mathrm{s}$ & $45^{\circ}$ \\
Parameterization II & $1 . d v_{p 1}$ & $2 . d v_{s 1}$ & $3 . d \varepsilon_{1}$ & $4 . d \varepsilon_{D}$ & $5 . d \eta_{1}$ & $6 . d \eta_{D}$ & $7 . d \delta_{3}$ & $8 . d \gamma_{D}$ & $9 . d \gamma_{1}$ & $10 . d \Phi$ \\
& $0.2 \mathrm{~km} / \mathrm{s}$ & $0.115 \mathrm{~km} / \mathrm{s}$ & 0.105 & 0.105 & 0.105 & 0.105 & 0.105 & -0.087 & 0.105 & $45^{\circ}$ \\
\hline
\end{tabular}

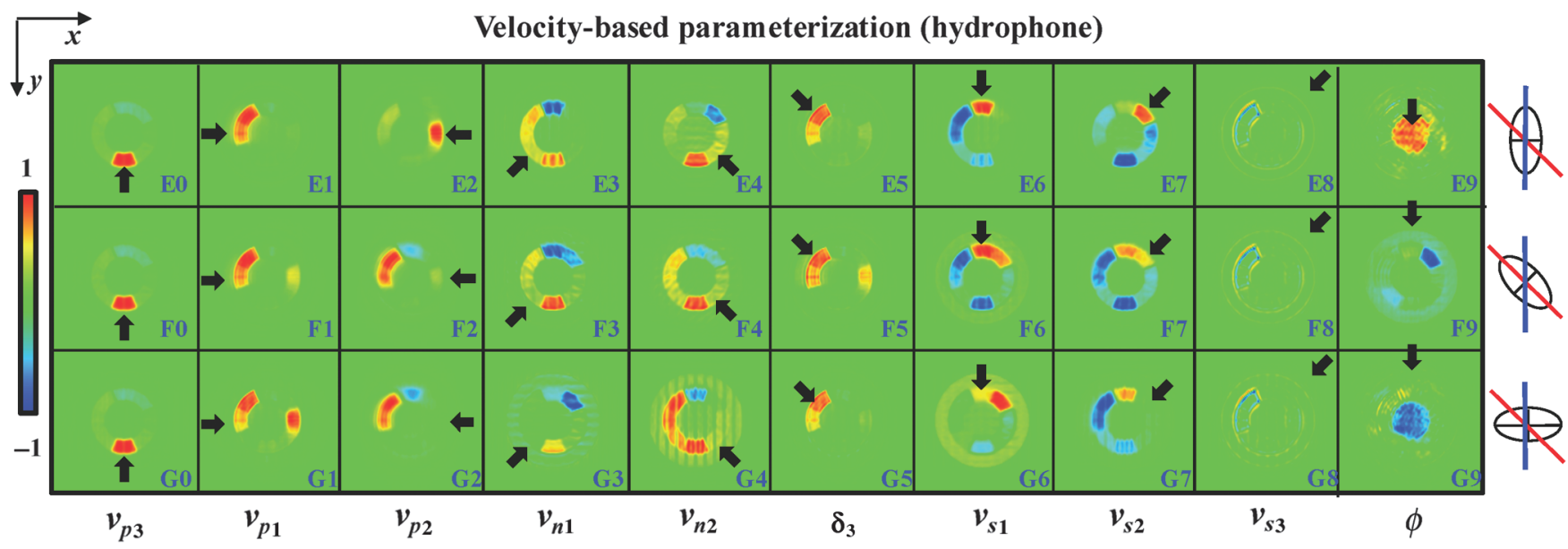

Figure 8. The horizontal slices of the gradient direction on the top of the hockey puck for each parameter using the velocity-based parameterization. We assume pressure sources and hydrophone data on the water surface. Each row shows the results from three different rotation angles $\left(0^{\circ}, 45^{\circ}\right.$, and $\left.90^{\circ}\right)$ in the inversion model. In the end of each row, the red and blue lines indicate the true fast axes of ORT media in the inner part (area 10) and the outer part (from area 1 to 9), respectively. The ellipsoid denotes the initial guess for the ORT axis in the whole puck (from area 1 to 10$)$. The black arrow indicates the true location of each parameter. 
apart from the true angle, respectively. As we expected, data fitting for each parameter except $v_{p 3}$ and $v_{s 3}$ is conducted along the wrong azimuth direction.

On the other hand, the hierarchical parameterization shows interesting features (Figure 9). In the first row, we observe that only five ORT parameters $\left(v_{p 1}, v_{s 1}, \varepsilon_{1}, \varepsilon_{D}\right.$, and $\left.\delta_{3}\right)$ and the rotation angle are sensitive to the streamer data. Between these parameters, trade-off also exists. The P-wave velocity (E0) is well-isolated, whereas $v_{s 1}$ (E1) and $\varepsilon_{1}$ (E2) suffer from strong trade-off with $v_{p 1}$. Due to the lack of diving waves in this example, $\varepsilon_{D}$ and $\boldsymbol{\delta}_{3}$, which depend often on diving waves, have relatively weak sensitivity. However, $\varepsilon_{D}$ is well-decoupled from $\varepsilon_{1}$, whereas it is slightly coupled with $\boldsymbol{\delta}_{3}$ (E6). All four parameters are strongly coupled with $v_{p 1}$. This observation indicates that the misfit due to $v_{p 1}$ perturbation should be resolved in the early stages; otherwise, considerable trade-off errors may be induced. The other three parameters, $\left(\eta_{1}, \eta_{D}\right.$, and $\left.\gamma_{D}\right)$ are strongly coupled with $v_{s 1}$, and the data are insensitive to parameter $\gamma_{1}$, as we also observed in the radiation pattern of $\gamma_{1}$ (Figure 4). In the second and third rows, we observe that five parameters $\left(v_{p 1}, v_{s 1}\right.$, $\varepsilon_{1}, \eta_{1}$, and $\gamma_{1}$ ) are independent of the background rotation angle. The convergence of the deviation parameters depends on the background rotation angle. However, at the last stage of the proposed multistage approach, the convergence of the deviation parameters can be improved by inverting for the rotation angle with those parameters simultaneously. are listed in Table 4. For the rotation angle in layer 6, we assume two different structures (cases-I and -II in Figure 10) to verify the trade-off of the rotation angle with other ORT parameters. The synthetic rotated elastic ORT data are acquired by fourth-order standard staggered grid finite-difference method (Graves, 1996), with the Ricker wavelet that has a peak frequency of $5 \mathrm{~Hz}$. Domain decomposition (Bohlen, 2002) is applied, in which the modeling process is parallelized over shots and subdomains, simultaneously. The convolution perfectly matched layer (Roden and Gedney, 2000) is applied to absorb artificial reflected waves from the model boundaries. To avoid storing source wavefields $u^{s}$ in equation 12 , we choose a time-frequency hybrid-domain approach (Sirgue et al., 2008), in which forward $u^{s}$ and backward $u^{r}$ wavefields are transformed to the frequency domain on the fly. The frequency band used in FWI is from 3 to $10 \mathrm{~Hz}$. We consider a total of 6400 pressure sources for the synthetic survey. However, we randomly chose only 100 sources at each iteration to reduce the computational cost (Warner et al., 2013). In total, 160,000 hydrophones are placed on the water surface. As an initial model (Figure 11), we use a smoothed VTI model for $v_{p 1}, v_{s 1}$, and $\varepsilon_{1}$. This is because, based on the radiation pattern (Figure 4), we do not expect to obtain smoothed updates for $v_{s 1}$ and $\varepsilon_{1}$ from FWI using the hierarchical parameterization. The initial $\eta_{1}$ is the same as the initial $\varepsilon_{1}$. The other six parameters, including the rotation angle, are set to zero.

\section{NUMERICAL EXAMPLE: SYNTHETIC MARINE STREAMER DATA}

To support our observations in the radiationpattern and trade-off analyses, we first compare the behavior of the velocity-based and hierarchical parameterizations on a more realistic 3D model. Then, we show the feasibility of the proposed multistage FWI on the noise-free rotated ORT data. For simplicity, we assume a simple 3D synthetic model (Figure 10) with a high-velocity mud-filled channel structure (layer 4) and fractured rotated ORT layer (layer 6) that resembles a carbonate reservoir. The true ORT parameters
Table 4. The model parameters of each layer in Figure 10.

\begin{tabular}{llllllllllc} 
& $v_{p 3}$ & $v_{s 1}$ & $\varepsilon_{1}$ & $\varepsilon_{2}$ & $\delta_{1}$ & $\delta_{2}$ & $\delta_{3}$ & $\gamma_{1}$ & $\gamma_{2}$ & Ref. \\
\hline Layer 1 & 1.5 & 0 & 0 & 0 & 0 & 0 & 0 & 0 & 0 & Water \\
Layer 2 & 2.0 & 1.15 & 0 & 0 & 0 & 0 & 0 & 0 & 0 & Isotropic \\
Layer 3 & 2.2 & 1.27 & 0.12 & 0.12 & 0.04 & 0.04 & 0 & 0.08 & 0.08 & VTI \\
Layer 4 & 3.4 & 1.63 & 0.1 & 0.1 & 0.03 & 0.03 & 0 & 0.05 & 0.05 & VTI \\
Layer 5 & 2.6 & 1.5 & 0.06 & 0.06 & 0.03 & 0.03 & 0 & 0.02 & 0.02 & VTI \\
Layer 6 & 2.8 & 1.7 & 0.07 & 0.15 & 0.03 & 0.08 & 0.02 & 0.04 & 0.08 & rORT \\
Layer 7 & 3.5 & 2.15 & 0.05 & 0.05 & 0.03 & 0.03 & 0 & 0.02 & 0.02 & VTI \\
Layer 8 & 4.5 & 2.9 & 0 & 0 & 0 & 0 & 0 & 0 & 0 & Isotropic \\
\hline
\end{tabular}

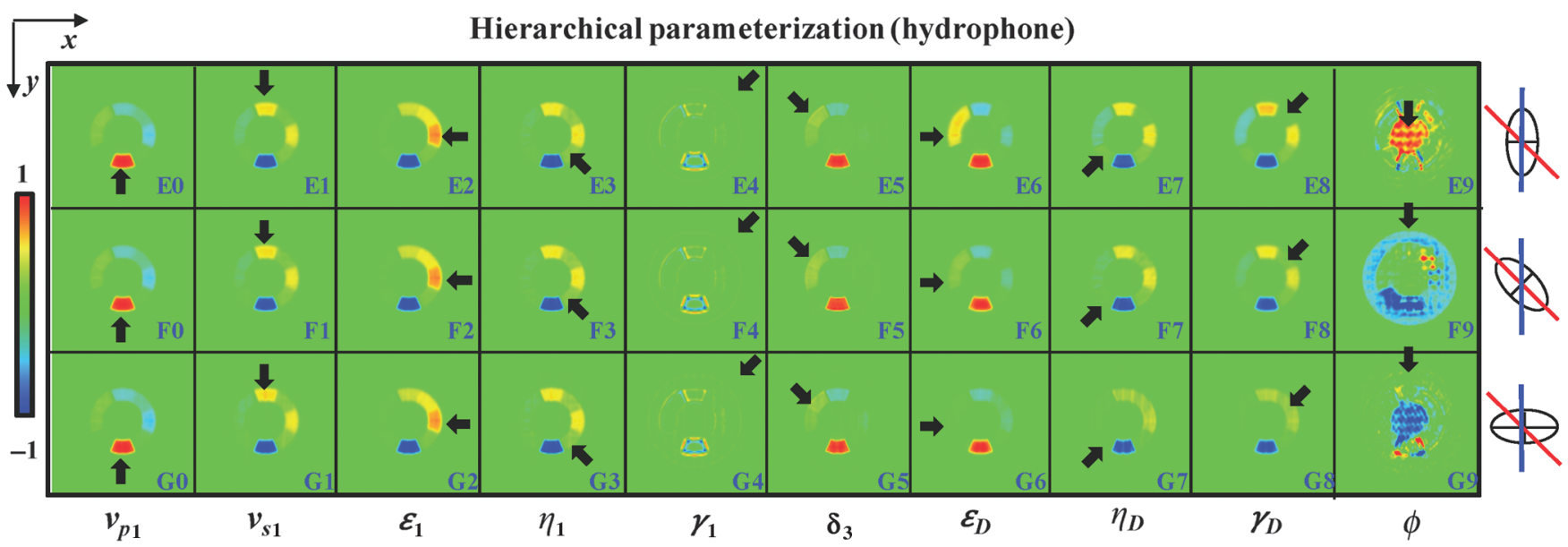

Figure 9. The same as Figure 8, but for the hierarchical parameterization. 
The true and initial densities are fixed at $1 \mathrm{~g} / \mathrm{cm}^{3}$ in the water and at $2 \mathrm{~g} / \mathrm{cm}^{3}$ in the layers beneath and not updated. The diagonal of the pseudoHessian matrix (Shin et al., 2001) is applied for ORT parameters whereas, for the rotation angle, we find the steepest descent solution to be preferable. As equation 31 shows, the virtual source as well as the gradient is controlled by the model parameter deviations along the fast and slow axes. This means that if the model deviation is too small close to VTI media, the virtual source will be very weak - almost zero, which means the pseudo-Hessian (zero-lag autocorrelation of the virtual source) will be also very small. For this reason, the gradient direction of the rotation angle can be extremely amplified by the pseudo-Hessian matrix when the model parameter deviation is very small at weak ORT areas. The FWI takes approximately $10 \mathrm{~min}$ per iteration with $100 \mathrm{compu}$ tational nodes on the Cray XC40.

Figure 12 shows the FWI results from the velocity-based parameterization. All 10 parameters are inverted simultaneously, and then they are converted to those in the hierarchical parameterization for comparison. Only the five main parameters, which are sensitive to marine streamer data, are displayed. Due to the poor initial guess for the rotation angle of the ORT anisotropy, the FWI using the velocity-based parameterization was not successful. The parameters $v_{p 1}$ and $v_{s 1}$ are relatively well-recovered, whereas $\varepsilon_{1}$ and $\varepsilon_{\mathrm{D}}$ are poorly inverted. Because we failed to recover background ORT parameters, the inversion for the rotation angle was also not successful.

Figure 13 shows the FWI results from the hierarchical parameterization. Following our observations in trade-off analysis, we only update five main parameters $\left(v_{p 1}, v_{s 1}, \varepsilon_{1}, \varepsilon_{D}\right.$, and $\left.\phi\right)$. In this simulation, all five parameters are inverted simultaneously. The FWI results are better than those from the velocity-based parameterization. However, it is clear that $\varepsilon_{1}$ and $\varepsilon_{D}$ suffer from the trade-off with the P-wave velocity because this channel-like imprint is obviously caused by the $\mathrm{P}$-wave velocity structure. The imprints from the $\mathrm{P}$-wave velocity error decrease $\varepsilon_{1}$ and increase $\varepsilon_{D}$, as also supported by the trade-off analysis in Figure 9 (E2 and E6). Due to the failure in inverting for $\varepsilon_{D}$, the inverted rotation angle is also not satisfactory. To reduce the trade-off, we apply the proposed multistage FWI strategy under the same FWI setting (Figure 14). Because the misfits in the data from $v_{p 1}$ are reduced first in an isotropic update stage, $\varepsilon_{D}$ structure is well-recovered in the following stage. From this good initial ORT assumption, the rotation angle is also well-recovered. Figure 15 shows the FWI results with the multistage strategy, but for the case-II rotation angle structure. As also supported by

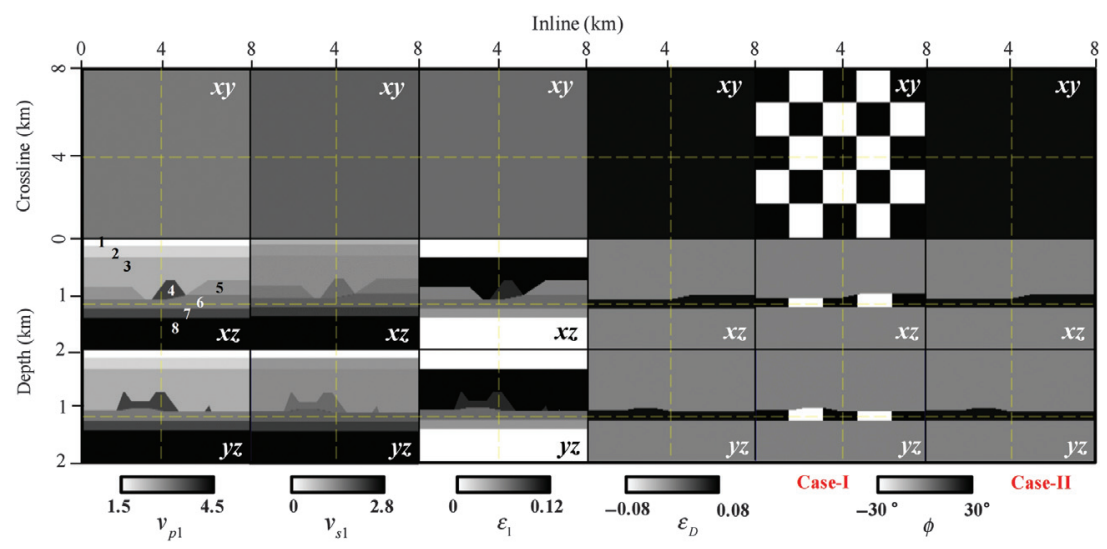

Figure 10. The true model parameters for the synthetic example. Note that we assume two different azimuth angle structures (cases-I and -II). The other five ORT parameters are listed in Table 4.

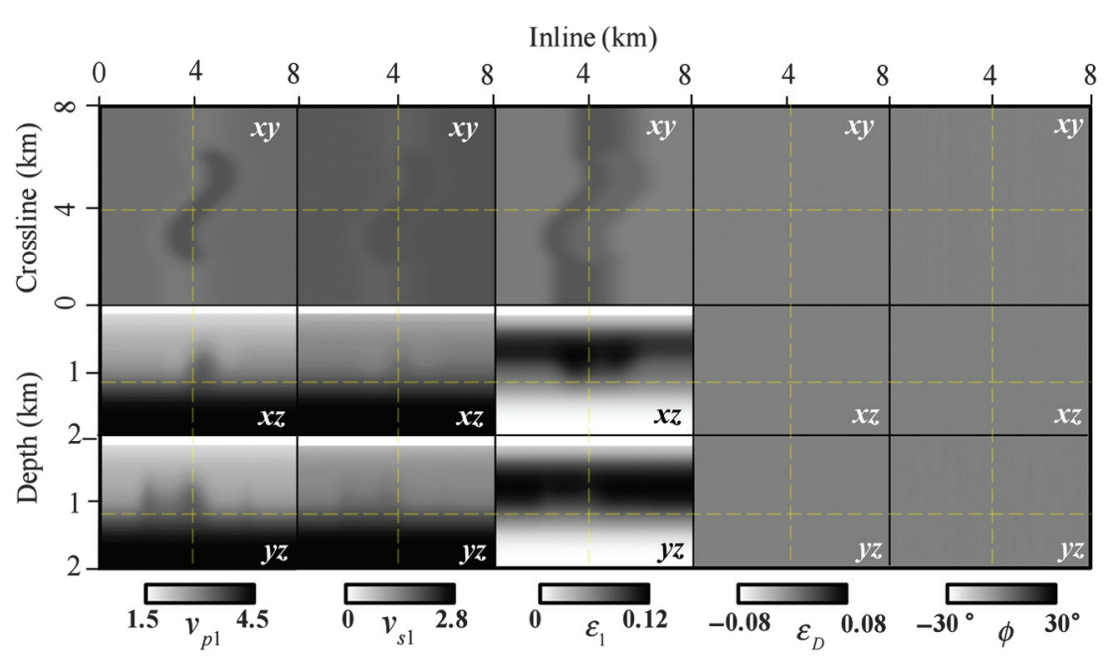

Figure 11. The smoothed initial VTI model. The initial $\eta_{1}$ is same as $\varepsilon_{1}$ and the other four ORT parameters are set to zero.

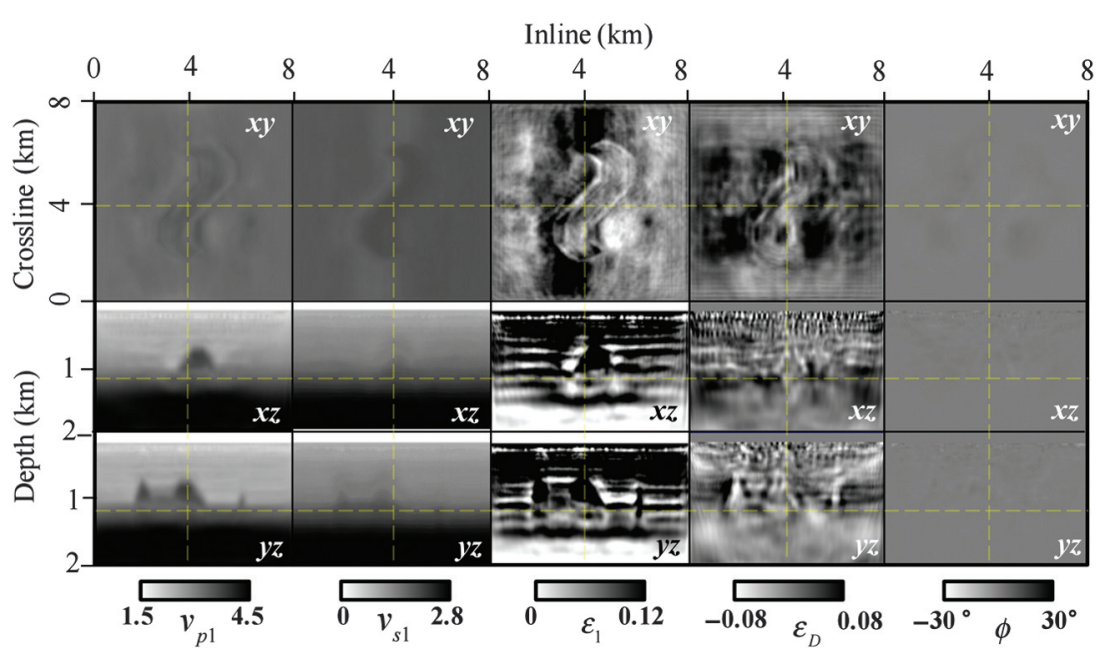

Figure 12. The final inverted model for case-I data using the velocity-based parameterization. Note that all 10 parameters in the velocity-based parameterization are inverted simultaneously and then converted to those in the hierarchical parameterization for comparison. Only five parameters are displayed. 


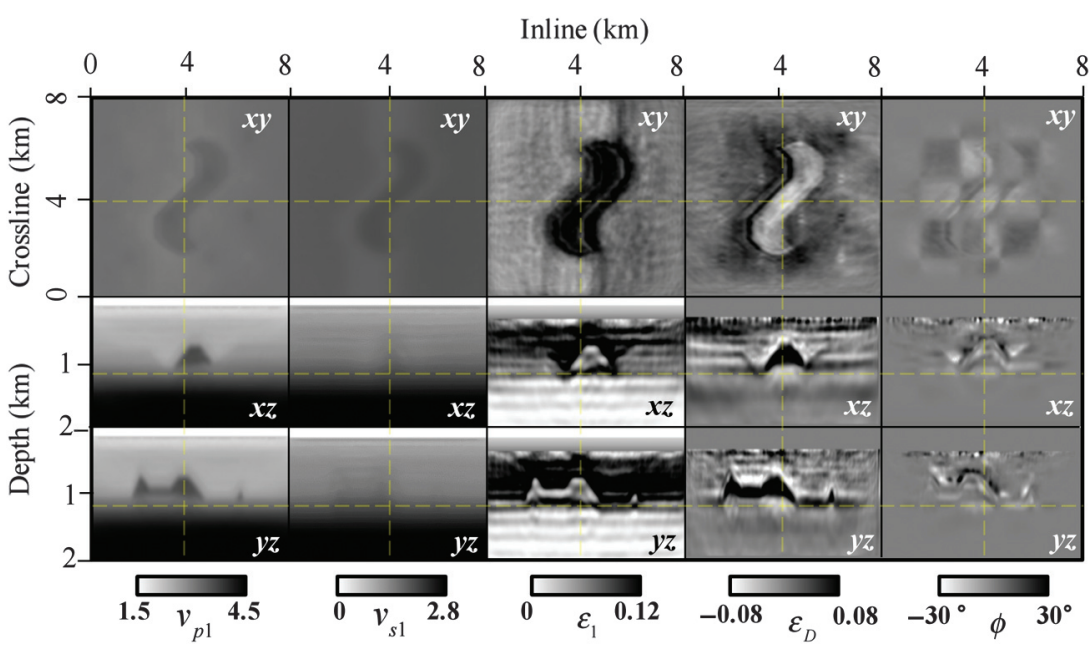

Figure 13. The final inverted model for case-I data using the hierarchical parameterization. Note that only five parameters are inverted simultaneously. Other five parameters are fixed during FWI.

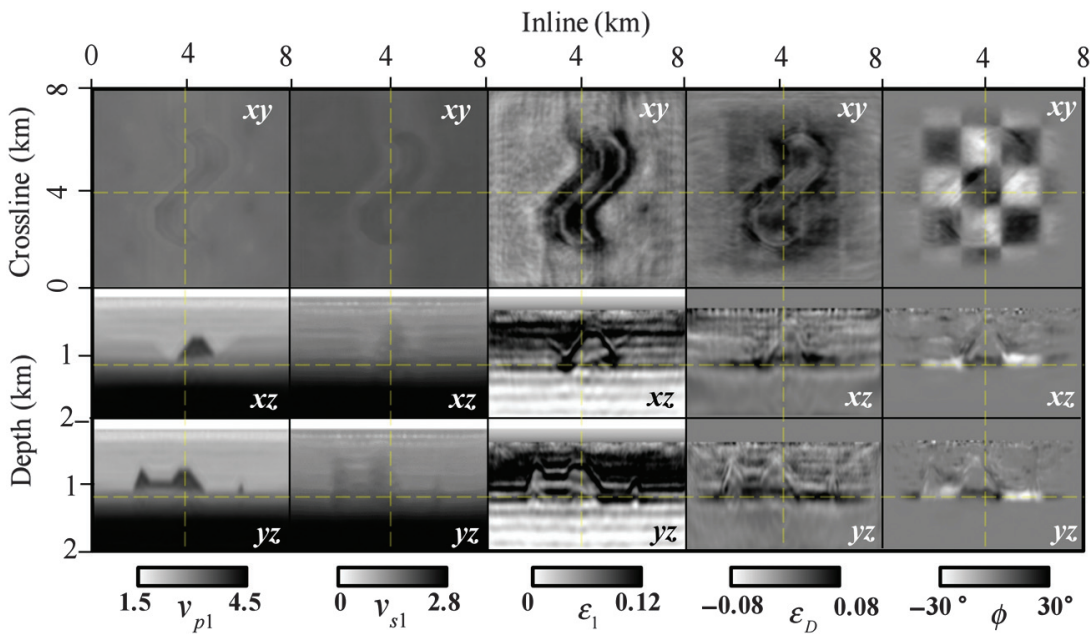

Figure 14. The final inverted model for case-I data using the hierarchical parameterization with the proposed multistage approach. Note that only five parameters are inverted from isotropic $\left(v_{p 1}\right.$ and $\left.v_{s 1}\right)$ to rotated ORT parameters by adding $\varepsilon_{1}, \varepsilon_{\mathrm{D}}$, and $\phi$, sequentially.

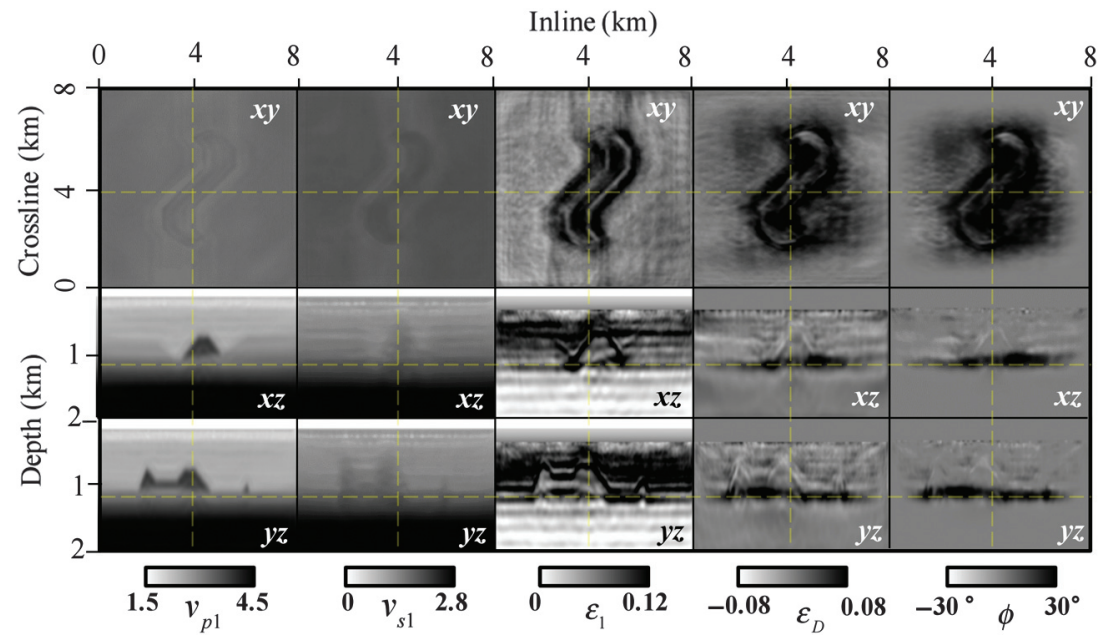

Figure 15. Same as Figure 14, but for case-II data. radiation-pattern and trade-off analyses, VTI parameters $v_{p 1}, v_{s 1}$, and $\varepsilon_{1}$ are recovered regardless of the rotation angle of ORT anisotropy. From this inverted VTI model, $\varepsilon_{D}$ and the rotation angle are well-recovered with less trade-off each other.

\section{CONCLUSION}

Assuming azimuthally rotated elastic ORT media, we examine the possibility of inverting for the rotation angle of the symmetric axis as a FWI target. To do that, we formulate the gradient direction and radiation pattern of the PDW using the elastic wave equation for monoclinic media. Then, we parameterize the monoclinic elastic wave equation with the velocity-based and hierarchical parameterizations. By analyzing the radiation patterns and the gradient directions of each parameterization, we are convinced that the hierarchical parameterization, in which we use deviation parameters, provides us with the best functionality to perform FWI in rotated ORT media. In the velocity-based parameterization, most parameters depend on the azimuth direction of the ORT anisotropy and thus, the azimuth direction of the data, which means that this parameterization requires accurate information on the azimuth angle. On the other hand, thanks to the separation of the scattering potentials in the hierarchical parameterization, we build the subsurface model from simple to complex anisotropy with less trade-off with the azimuthal rotation. Trade-off analysis for the hockey-puck-shaped model clearly shows the sensitivity of each parameter, and as a result, we realized that we could mainly estimate five ORT parameters from marine streamer data using the hierarchical parameterization. The numerical examples show that a multistage FWI implementation reduces the trade-off between parameters so that, finally, the azimuth angle can be estimated.

\section{ACKNOWLEDGMENTS}

Research reported in this publication was supported by competitive research funding from King Abdullah University of Science and Technology (KAUST) in Thuwal, Saudi Arabia. This research was also supported by Basic Science Research Program through the National Research Foundation of Korea (NRF) funded by the Ministry of Science and ICT (NRF2017R1C1B5077123). For computer time, this research used the resources of the Supercomputing Laboratory in KAUST. We also thank the members of Seismic Wave Analysis Group in KAUST for helpful discussion. We would like to thank K. Innanen as the associate editor, J. Chen, J. Hu, and one anonymous reviewer for their helpful suggestions. 


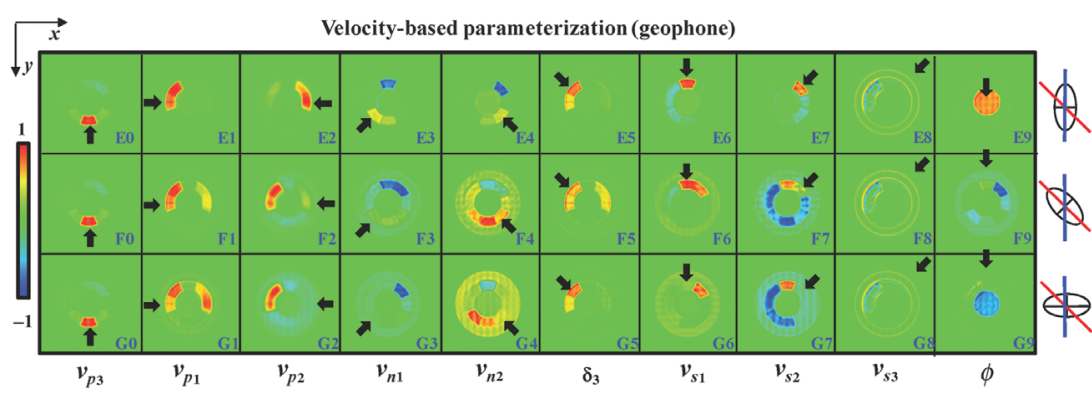

Figure A-1. The same as Figure 8, but for the 3C geophone data on the sea bottom.

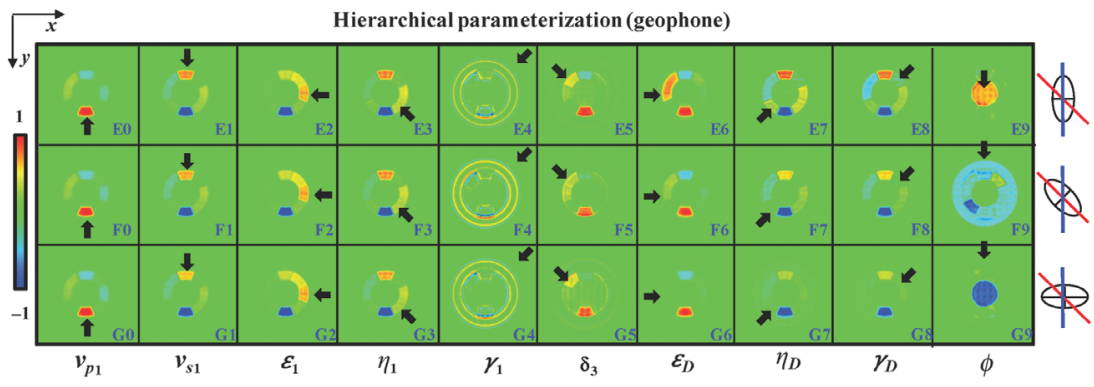

Figure A-2. The same as Figure 9, but for the 3C geophone data on the sea bottom.

Alkhalifah, T., 2016, Research note: Insights into the data dependency on anisotropy: An inversion prospective: Geophysical Prospecting, 64, 505-513, doi: $10.1111 / 1365-2478.12345$

Alkhalifah, T., N. Masmoudi, and J. W. Oh, 2016, A recipe for practical full waveform inversion in orthorhombic anisotropy: The Leading Edge, 35, 10761083, doi: 10.1190/tle35121076.1.

Alkhalifah, T., and R.-E. Plessix, 2014, A recipe for practical full-waveform inversion in anisotropic media: An analytical parameter resolution study: Geophysics, 79, no. 3, R91-R101, doi: 10.1190/ geo2013-0366.1.

Backus, G. E., 1962, Long-wave elastic anisotropy produced by horizontal layering: Journal of Geophysical Research, 67, 4427-4440, doi: 10.1029/ JZ067i011p04427.

Bai, J., and D. Yingst, 2014, Simultaneous inversion of velocity and density in time-domain full waveform inversion: 84th Annual International Meeting, SEG, Expanded Abstracts, 922-927.

Bakulin, A., V. Grechka, and I. Tsvankin, 2000, Estimation of fracture parameters from reflection seismic data. Part 1: HTI model due to a single fracture set: Geophysics, 65, 1788-1802, doi: 10.1190/1 .1444863 .

Bohlen, T., 2002, Parallel 3-D viscoelastic finite difference seismic modeling: Computers \& Geosciences, 28, 887-899, doi: 10.1016/S0098-3004(02)00006-7.

Brossier, R., S. Operto, and J. Virieux, 2009, Seismic imaging of complex onshore structures by $2 \mathrm{D}$ elastic frequency-domain full-waveform inversion: Geophysics, 74, no. 6, WCC105-WCC118, doi: 10 $.1190 / 1.3215771$

Downton, J., and B. Roure, 2010, Azimuthal simultaneous elastic inversion for fracture detection: 80th Annual International Meeting, SEG, Expanded

APPENDIX A

\section{TRADE-OFF ANALYSIS FOR OBC ACQUISITION}

In Appendix A, we show the trade-off analysis based on oceanbottom cable (OBC) acquisition. For the same FWI setting for the hockey-puck model (Figure 7), 160,000 3C geophones (displacement fields) are placed on the sea bottom. Figures A-1 and A-2 show the trade-off patterns from the OBC acquisition system. Unlike for the streamer data (Figures 8 and 9), the $\mathrm{P}$ and $\mathrm{S}$-wave reflections are available in OBC data (Raknes and Arntsen, 2014). Thanks to S-waves, more parameters are sensitive to OBC data and resolution is improved. For the velocity-based parameterization, the recoverability of each parameter is reasonably improved when the exact azimuth angle of the ORT medium is known (the first row in Figure A-1). However, this parameterization still requires a good estimation of such an azimuth angle. The recoverability of each parameter in the hierarchical parameterization is also improved (Figure A-2). However, three parameters $\left(\eta_{1}, \eta_{\mathrm{D}}\right.$, and $\gamma_{\mathrm{D}}$ ) are still weakly recovered and are strongly coupled with $v_{s 1}$. The parameter $\gamma_{1}$ is not sensitive even to OBC data, as supported by the radiation pattern (Figure 4 ) and the influence of $\boldsymbol{\delta}_{3}$ is weaker than $\varepsilon_{\mathrm{D}}$. For this reason, five parameters $\left(v_{p 1}, v_{s 1}, \varepsilon_{1}, \varepsilon_{\mathrm{D}}\right.$, and $\left.\phi\right)$ in the hierarchical parameterization are still considered as the main parameters for OBC data (Oh and Alkhalifah, 2016a).

\section{REFERENCES}

Aki, K., and R. G. Richards, 1980, Quantitative seismology: Freeman. Albertin, U., P. Shen, A. Sekar, T. Johnsen, C. Wu, K. Nihei, and K. Bube, 2016, 3D orthorhombic elastic full-waveform inversion in the reflection domain from hydrophone data: 86th Annual International Meeting, SEG, Expanded Abstracts, 1094-1098.

Alkhalifah, T., 2003, An acoustic wave equation for orthorhombic anisotropy: Geophysics, 68, 1169-1172, doi: 10.1190/1.1598109.
Abstracts, 263-267, doi: 10.1190/1.3513389.

Gholami, Y., R. Brossier, S. Operto, A. Ribodetti, and J. Virieux, 2013 Which parameterization is suitable for acoustic vertical transverse isotropic full waveform inversion? Part 1: Sensitivity and trade-off analysis: Geophysics, 78, no. 2, R81-R105, doi: 10.1190/geo2012-0204.1.

Graves, R. W., 1996, Simulating seismic wave propagation in 3D elastic media using staggered-grid finite differences: Bulletin of the Seismological Society of America, 86, 1091-1106.

Guitton, A., and T. Alkhalifah, 2016, Full-waveform inversion in an anisotropic elastic earth: Can we isolate the role of density and shear wave velocity? 78th Annual International Conference and Exhibition, EAGE, Extended Abstracts, doi: 10.3997/2214-4609.201601193.

He, W., and R.-E. Plessix, 2016, Analysis of different parameterizations of waveform inversion of compressional body waves in an elastic transverse isotropic earth with a vertical axis of symmetry: Geophysical Prospecting, 65, 1004-1024, doi: 10.1111/1365-2478.12452.

Ivanov, Y., and A. Stova, 2016, Upscaling in orthorhombic media: Behavior of elastic parameters in heterogeneous fractured earth: Geophysics, 81, no. 3, C113-C126, doi: 10.1190/geo2015-0392.1.

Kamath, N., and I. Tsvankin, 2016, Elastic full-waveform inversion for VTI media: Methodology and sensitivity analysis: Geophysics, 81, no. 2, C53-C68, doi: 10.1190/geo2014-0586.1.

Köhn, D., O. Hellwig, D. D. Nil, and W. Rabbel, 2015, Waveform inversion in triclinic anisotropic media: A resolution study: Geophysical Journal International, 201, 1642-1656, doi: 10.1093/gji/ggv097.

Köhn, D. D. D. Nil, A. Kurzmann, A. Przebindowska, and T. Bohlen, 2012, On the influence of model parametrization in elastic full waveform tomography: Geophysical Journal International, 191, 325-345, doi: 10 $.1111 / \mathrm{j} .1365-246 X .2012 .05633 . x$.

Ma, Y., M. Maharramov, R. Clapp, and B. Biondi, 2016, Multiparameter full-waveform inversion in the isotropic acoustic media with application to time-lapse seismic inverse problem: 86th Annual International Meeting, SEG, Expanded Abstracts, 5557-5561.

Masmoudi, N., and T. Alkhalifah, 2016, A new parameterization for waveform inversion in acoustic orthorhombic media: Geophysics, 81, no. 4 R157-R171, doi: 10.1190/geo2015-0635.1.

Masmoudi, N., A. Stovas, and T. Alkhalifah, 2017, Scanning anisotropy parameters in horizontal transversely isotropic media: Geophysical Prospecting, 65, 981-991, doi: 10.1111/1365-2478.12464.

Oh, J. W., and T. Alkhalifah, 2016a, Elastic orthorhombic anisotropic parameter inversion: An analysis of parameterization: Geophysics, 81, no. 6, C279-C293, doi: 10.1190/geo2015-0656.1.

Oh, J. W., and T. Alkhalifah, 2016b, 3D elastic-orthorhombic anisotropic full-waveform inversion: Application to field OBC data: 86th Annual International Meeting, SEG, Expanded Abstracts, 1206-1210. 
Oh, J. W, and T. Alkhalifah, 2018, Full waveform inversion using envelopebased global correlation: Geophysical Journal International, 213, 815823, doi: $10.1093 / \mathrm{gji} / \mathrm{ggy} 031$.

Oh, J. W., T. Alkhalifah, and D. J. Min, 2015, Multi-stage full waveform inversion strategy for 2D elastic VTI media: 85th Annual International Meeting, SEG, Expanded Abstracts, 1204-1208.

Oh, J. W., M. Kalita, and T. Alkhalifah, 2018, 3D elastic full waveform inversion using P-wave excitation amplitude: Application to ocean bottom cable field data: Geophysics, 83, no. 2, R129-R140, doi: 10.1190/ geo2017-0236.1.

Operto, S. Y., Y. Gholami, V. Prieux, A. Ribodetti, R. Brossier, L. Métivier, and J. Virieux, 2013, A guided tour of multiparameter full-waveform inversion with multicomponent data: From theory to practice: The Leading Edge, 32, 1040-1054, doi: 10.1190/tle32091040.1.

Pan, W., K. A. Innanen, G. F. Margrave, M. C. Fehler, X. Fang, and J. Li, 2016, Estimation of elastic constants for HTI media using Gauss-Newton and full-Newton multiparameter full-waveform inversion: Geophysics, 81, no. 5, R275-R291, doi: 10.1190/geo2015-0594.1.

Pratt, R. G., C. Shin, and G. Hick, 1998, Gauss-Newton and full Newton methods in frequency-space seismic waveform inversion: Geophysical Journal International, 133, 341-362, doi: 10.1046/j.1365-246X.1998 .00498.x.

Prieux, V., R. Brossier, S. Operto, and J. Virieux, 2013, Multiparameter full waveform inversion of multicomponent ocean-bottom-cable data from the Valhall field. Part 2: Imaging compressive wave and shear-wave velocities: Geophysical Journal International, 194, 1665-1681, doi: 10.1093/ gji/ggt178.

Raknes, E. B., and B. Arntsen, 2014, Strategies for elastic full waveform inversion: 84th Annual International Meeting, SEG, Expanded Abstracts, $1222-1226$.

Raknes, E. B., and W. Weibull, 2016, Efficient 3D elastic full-waveform inversion using wavefield reconstruction methods: Geophysics, 81, no. 2, R45-R55, doi: 10.1190/geo2015-0185.1.

Roden, J. A., and S. D. Gedney, 2000, Convolution PML (CPML): An efficient FDTD implementation of the CFS-PML for arbitrary media: Microwave and Optical Technology Letters, 27, 334-339, doi: 10.1002/(ISSN) $1098-2760$.

Rusmanugroho, H., R. Modrak, and J. Tromp, 2017, Anisotropic full-waveform inversion with tilt-angle recovery: Geophysics, 82, no. 3, R135R151, doi: 10.1190/geo2016-0025.1.
Shin, C., S. Jang, and D. J. Min, 2001, Improved amplitude preservation for prestack depth migration by inverse scattering theory: Geophysical Prospecting, 49, 592-606, doi: 10.1046/j.1365-2478.2001.00279.x.

Silva, N. V., A. Ratcliffe, V. Vinje, and G. Conroy, 2016, A new parameter set for anisotropic multiparameter full-waveform inversion and application to a North Sea data set: Geophysics, 81, no. 4, U25-U38, doi: 10 $.1190 /$ geo2015-0349.1.

Sirgue, L., J. Etgen, and U. Albertin, 2008, 3D frequency domain waveform inversion using time domain finite difference methods: 70th Annual International Conference and Exhibition, EAGE incorporating SPE EUROPEC 2008, Extended Abstracts, doi: 10.3997/2214-4609.20147683.

Stovas, A., 2015, Azimuthally dependent kinematic properties of orthorhombic media: Geophysics, 80, no. 6, C107-C122, doi: 10.1190/geo2015-0288.1.

Tarantola, A., 1986, A strategy for nonlinear elastic inversion of seismic reflection data: Geophysics, 51, 1893-1903, doi: 10.1190/1.1442046.

Thomsen, L., 1986, Weak elastic anisotropy: Geophysics, 51, 1954-1966, doi: 10.1190/1.1442051.

Tsvankin, I., 1997, Anisotropic parameters and P-wave velocity for orthorhombic media: Geophysics, 62, 1292-1309, doi: 10.1190/1.1444231.

Tsvankin, I., 2001, Seismic signatures and analysis of reflection data in anisotropic media: Elsevier Science.

Tsvankin, I., 2012, Seismic signatures and analysis of reflection data in anisotropic media, 3rd ed.: SEG.

Vireiux, J., and S. Operto, 2009, An overview of full-waveform inversion in exploration geophysics: Geophysics, 74, no. 6, WCC1-WCC25, doi: 10 $.1190 / 1.3238367$

Wang, H., and I. Tsvankin, 2016, Feasibility of waveform inversion in acoustic orthorhombic media: 86th Annual International Meeting, SEG, Expanded Abstracts, 311-316.

Warner, M.. A. Ratcliffe, T. Nangoo, J. Morgan, A. Umpleby, N. Shah, V. Vinje, I. Štekl, L. Guasch, and C. Win, 2013, Anisotropic 3D full-waveform inversion: Geophysics, 78, no. 2, R59-R80, doi: 10.1190/geo2012-0338.1.

Xie, Y., B. Zhou, J. Zhou, J. Hu, L. Xu, X. Wu, N. Lin, F. C. Loh, L. Liu, and Z. Wang, 2017, Orthorhombic full-waveform inversion for imaging the Luda field using wide-azimuth ocean-bottom-cable data: The Leading Edge, 36, 75-80, doi: 10.1190/tle36010075.1.

Yin, D., S. Z. Sun, P. Yang, and L. Peng, 2013, Influence of seismic acquisition on AVOZ inversion: A case study in Ha7 area of Tarim Basin: 83rd Annual International Meeting, SEG, Expanded Abstracts, 3180-3184, doi: 10.1190/segam2013-1315.1 